\title{
User association and channel assignment in downlink multi-cell NOMA networks: A matching-theoretic approach
}

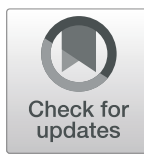

Mohammed W. Baidas ${ }^{1 *}$ (D), Zainab Bahbahani² and Emad Alsusa ${ }^{3}$

\begin{abstract}
This paper studies the problem of stable user association and channel assignment in downlink multi-cell non-orthogonal multiple-access (NOMA) networks. To be specific, the goal is to assign network users to the channels at each base station, while accounting for inter-user interference and maintaining quality of service (QoS) per user. To that end, a low-complexity iterative solution procedure is devised to obtain the optimal power allocation for proportional fairness signal-to-interference-plus-noise ratio (SINR)-based maximization, which is then utilized to determine the preferences of network users over the channels available at each base station and the preferences of base stations over the network users. In turn, a many-to-one matching-theoretic model based on the student-project allocation problem is applied. Particularly, two polynomial-time complexity stable matching algorithms are proposed to associate users with base stations and perform channel assignment, such that no user or base station would deviate and change its association or channel assignment unilaterally. To validate the efficacy of the proposed solution procedure and stable matching algorithms, extensive simulation results are presented to compare them to a centralized joint user association, channel assignment, and power allocation (C-J-UA-CA-PA) scheme. It is demonstrated that the proposed algorithms efficiently associate users with base stations and assign them to channels as well as efficiently yielding comparable SINR per user to the C-J-UA-CA-PA scheme, while maximizing proportional fairness and satisfying QoS constraints.
\end{abstract}

Keywords: Channel assignment, Matching, Multi-cell, Non-orthogonal multiple access, Proportional fairness, User association

\section{Introduction}

Non-orthogonal multiple access (NOMA) has recently attracted so much attention to meet the ever-increasing demand for high spectral efficiency, improved fairness, massive connectivity, low transmission latency, and high throughput in future 5G cellular networks [1]. Specifically, the principle of NOMA is based on multiplexing users in the power domain over resource blocks by enforcing power imbalance between the transmitted user signals, which allows successive interference cancelation (SIC) to effectively eliminate inter-user interference, and yielding considerable performance gains over conventional orthogonal multiple access (OMA) schemes [2]. To date,

\footnotetext{
*Correspondence: m.baidas@ku.edu.kw
}

${ }^{1}$ Department of Electrical Engineering, College of Engineering and Petroleum, Kuwait University, Kuwait City, Kuwait

Full list of author information is available at the end of the article the majority of the published literature on resource allocation for NOMA systems has considered on single-cell networks. For example, the problem of sum-throughput maximizing power allocation based on $\alpha$-fairness in single-cell downlink NOMA networks is studied in [3]. Particularly, the cases of statistical and perfect channel state information at the transmitter (CSIT) are considered. For the former case, fixed target data rates are pre-defined for all users, while for the latter case, users' rates are adapted according to the instantaneous CSI. In [4], the authors propose user-pairing schemes for capacity maximization in single-cell downlink NOMA networks. To be specific, two user-pairing schemes have been proposed so as to provide capacity gains to almost all users by grouping them in pairs. Additionally, the exact sum capacity of a two-user pair has been analytically derived, while taking into account perfect and imperfect SIC. A 
dynamic power allocation scheme-subject to quality-ofservice requirements-for downlink and uplink single-cell NOMA networks with two users has been considered in [5]. Moreover, the exact expressions for the outage probability and average rate resulting from the proposed scheme are obtained. The problem of optimal power allocation with given channel assignment under different performance criteria for single-cell downlink NOMA networks is studied in [6]. Furthermore, the authors proposed a low-complexity scheme for joint channel assignment and power allocation via a dynamic/iterative matching algorithm. Although single-cell NOMA networks have received significant attention in the past few years, much less attention has been given to multi-cell NOMA networks. Therefore, it is of paramount importance to study NOMA in the more realistic multi-cell scenario, which is much more challenging due to the complex interplay between multiple cells [7].

Recently, a few research works have focused on resource allocation in multi-cell NOMA networks. For example, in [8], distributed power control for downlink multicell NOMA networks is studied. Particularly, the authors study the problem of total transmit power minimization of all base stations, subject to minimum data rate requirements of network users. Moreover, a distributed algorithm is devised, which has been shown to converge to the optimal solution, if one exists. The problems of sum-power minimization (SPM) and sum-rate maximization (SRM) for multi-cell NOMA networks are studied in [9]. To be specific, the SPM is transformed into a linear programming problem, and closed-form solutions to the power allocation of each user are determined. Contrarily, the SRM problem is solved by decomposing it into a power allocation problem for users in a single cell, and a power control problem over multiple cells. After obtaining the optimal sum rate in a single cell, a distributed algorithm is devised to solve the original sum-rate maximization. In [10], the authors consider the problem of jointly optimizing power allocation, user-pair selection, and time-frequency resource allocation in multi-cell NOMA networks. In particular, an efficient algorithm for obtaining the equilibrium for resource allocation-while taking into account inter-cell interference-is proposed, which has also been proven to be the global optimum resource allocation solution. In [11], the authors outline a general framework for coordinated multipoint (CoMP) transmission in downlink multi-cell NOMA systems with distributed power allocation at each cell. Specifically, the applicability and necessary conditions for different CoMP schemes and network scenarios are investigated, and simulation results are presented to quantify the spectral efficiency gains of CoMP-NOMA over CoMP-OMA. Efficient power allocation for sum network capacity maximization in downlink multi-cell multi-user NOMA networks is considered in [12]. Particularly, a local optimal solution iterative scheme is proposed, which has been shown to outperform non-optimal NOMA and OMA schemes. In [13], the performance of NOMA in multi-cell downlink millimeter-wave (mmWave) networks is investigated. To be specific, closed-form outage probability expressions are derived, where it has been demonstrated that NOMA can outperform OMA in multi-cell mmWave networks.

This paper studies the problem of stable user association and channel assignment in downlink multi-cell NOMA networks. To be specific, the goal is to assign network users to the channels available at each base station, while accounting for inter-user interference, and maintaining quality of service (QoS) per user. To that end, a lowcomplexity iterative solution procedure-that can be executed locally at each base station-is devised to obtain the optimal power allocation for proportional fairness signalto-interference-plus noise ratio (SINR)-based maximization, which is utilized to determine the preferences of network users over the channels available at each base station, and the preferences of base stations over the network users. In turn, a many-to-one matching-theoretic model based on the student-project allocation (SPA) problem is applied [14, 15]. Particularly, two polynomial-time complexity algorithms are proposed, namely the user-oriented stable matching (U-SM) and the base station-oriented stable matching (BS-SM). The proposed SPA-based stable matching algorithms incorporate constraints on the number of users per channel and the number of users to be associated with each base station. Furthermore, in the U-SM algorithm, a user-optimal stable matching solution is achieved, while in the BS-SM algorithm, a base station-optimal stable matching solution is obtained. More importantly, the U-SM and BS-SM algorithms yield user association and channel assignment stable matchings that are simultaneously best response for all users and all base stations, respectively, such that no user or base station would deviate and change its association or channel assignment unilaterally. Additionally, a centralized joint user association, channel assignment, and power allocation (C-J-UA-CA-PA) optimization problem is formulated and shown to be computationally expensive. To validate the efficacy of the proposed solution procedure and stable matching algorithms, extensive simulation results are presented to compare them to the C-J-UACA-PA scheme. It is demonstrated that the proposed algorithms efficiently associate users with base stations and assign them to channels as well as yielding comparable SINR per user to the C-J-UA-CA-PA scheme, while maximizing proportional fairness and satisfying QoS constraints.

Few recent research works have applied matching theory to NOMA networks. For example, in [16], user pairing 
in a single-cell cognitive radio-inspired NOMA network is studied, where the base station allocates power to paired users within a cluster. In particular, a user with poor channel conditions is paired with a user with good channel conditions, while satisfying their rate requirements. To that end, a two-sided one-to-one distributed matching algorithm is developed, which is shown to yield performance that approaches that of centralized user pairing and power allocation. The authors in [17] investigate channel assignment, power allocation, and scheduling for single-cell downlink NOMA networks. To be specific, the problem of joint channel assignment and sum-rate maximizing power allocation with user fairness is considered. Moreover, a many-to-many userchannel matching algorithm is proposed, and an iterative solution for joint channel assignment and power allocation based on swap matching is devised. In [18], the authors consider the problem of sum-rate maximization for device-to-device (D2D) communication in uplink single-cell NOMA networks by optimizing channel and power allocation. In particular, a two-sided many-to-one matching algorithm is proposed to allow D2D groups to reuse the same channel occupied by a cellular user. Power allocation is also studied, where swap matching is applied to jointly allocate channel and power to the D2D groups. In [19], the problem of joint spectrum allocation and power control in single macro-cell NOMAenhanced heterogenous networks is considered. In particular, several many-to-one matching-theoretic algorithms with a swap operation are proposed while incorporating users' fairness in sum-rate maximizing power allocation. Clearly, all the aforementioned works focus on single-cell NOMA networks.

To the best of our knowledge, no prior work has applied the SPA matching problem for user association and channel assignment with proportional fairness SINR-based power allocation in downlink multi-cell NOMA networks. In turn, the main contributions of this work are summarized as follows:

- Proposed a low-complexity iterative solution procedure for determining the proportional fairness SINR-based maximizing power allocation for different user sets over the channels available at each base station.

- Modeled the user association and channel assignment problem in downlink multi-cell NOMA networks as a SPA matching problem.

- Devised two polynomial-time complexity stable matching algorithms based on the SPA matching problem, which associate network users with base stations and channels, such that user optimal and base station-optimal stable matching solutions are obtained.
- Compared the proposed algorithms to the C-J-UA-CA-PA scheme and demonstrated that the proposed algorithms yield comparable SINR per user as well as efficiently assigning channels to cell-edge users, while maximizing proportional fairness and satisfying QoS constraints.

The proposed algorithmic designs in this work aim at filling the gap for effective resource allocation solutions in multi-cell NOMA-based 5G cellular networks. In particular, the proposed solution procedure can be executed locally at each base station to efficiently determine the proportional fairness SINR-based maximizing power allocation of cellular users over each channel and within each base station, and with minimal computational complexity, while taking into account inter-user interference, QoS requirements, and SIC decoding constraints. In fact, the proposed solution procedure can incorporate other power allocation strategies and performance criteria (e.g., sum rate, energy efficiency, etc.) to establish the preferences of users over channels and base stations over users. Furthermore, the proposed stable matching algorithms can efficiently be executed among the base stations and without the need for a centralized controller. In summary, by optimizing the SINR of the network users via proportional fairness-based power allocation, and efficiently associating users with base stations and assigning channels to them over multiple cells, this work fulfills some of the requirements of NOMA-based 5G cellular networks.

The rest of this paper is organized as follows. Section 2 presents the network model, while Section 3 presents the centralized joint user association, channel assignment, and power allocation problem formulation. Section 4 discusses the proposed solution procedure for proportional fairness SINR-based maximizing power allocation. In Section 5, the SPA-based stable matching algorithms are devised, whereas in Section 6, the simulation results are presented. Finally, Section 7 draws the conclusions.

\section{Network model}

Consider a downlink multi-cell NOMA network with a set of $Q$ base stations (BSs), denoted $\mathcal{B}=\left\{\mathrm{BS}_{1}, \mathrm{BS}_{2}, \ldots, \mathrm{BS}_{Q}\right\}$, a set of $N$ users $\mathcal{U}=\left\{U_{1}, U_{2}, \ldots, U_{N}\right\}$, and a set of $K$ channels $\mathcal{C}=\left\{C_{1}, C_{2}, \ldots, C_{K}\right\}$. Particularly, the network bandwidth is divided into $K$ non-overlapping equal bandwidth channels, such that each base station $\mathrm{BS}_{q} \in \mathcal{B}$ is allocated a non-empty set of channels $\mathcal{C}_{q} \subset \mathcal{C}$, where $\mathcal{C}_{1}, \mathcal{C}_{2}, \ldots, \mathcal{C}_{q}$ partition $\mathcal{C}$. In other words, $\mathcal{C}_{q} \cap \mathcal{C}_{w}=\phi$ for $w \neq q$, and $\bigcup_{q=1}^{Q} \mathcal{C}_{q}=\mathcal{C}$. Furthermore, let $\mathcal{U}_{q} \subset \mathcal{U}$ be the subset of network users within the coverage area of each base station $\mathrm{BS}_{q} \in \mathcal{B}$ (see Fig. 1). Also, let $\zeta_{q, k}$ be the maximum number of users that can be assigned to channel $C_{q, k} \in \mathcal{C}_{q}$. That is, each channel $C_{q, k} \in \mathcal{C}_{q}$ can be occupied 


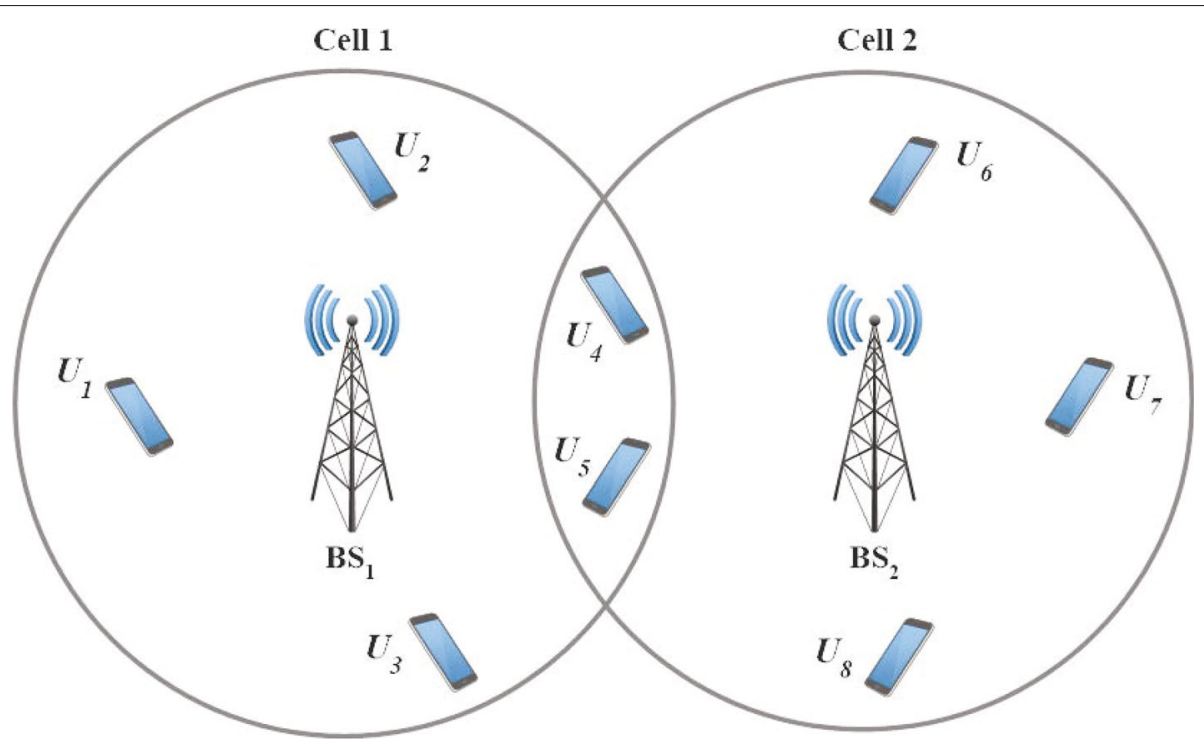

Fig. 1 A downlink multi-cell NOMA network with $Q=2$ base stations and $N=8$ users

by at most $\zeta_{q, k}$ users, $\forall B S_{q} \in \mathcal{B}^{1}$. Moreover, note that some network users may fall within the overlapping region of two or more cells. Therefore, the maximum number of users that can be associated with each base station $\mathrm{BS}_{q}$ is set to $\xi_{q}$ (i.e., a quota per base station). Additionally, each user may be associated with at most one base station and assigned to one channel. Our network model mimics that of a multi-cell orthogonal frequency-division multiple access (OFDMA)-NOMA network, where each channel is allocated to only one user, as per conventional OFDMA networks; while multiple users within a cell can share a channel via NOMA ${ }^{2}$.

In downlink NOMA networks, each base station $\mathrm{BS}_{q}$ sends data (over each assigned channel $C_{q, k} \in \mathcal{C}_{q}$ ) to its users simultaneously via power-domain superposition coding. The instantaneous channel between each BS and the network users within its coverage area follows narrowband Rayleigh fading with zero-mean $N_{0}$-variance additive white Gaussian noise ${ }^{3}$. Particularly, $h_{q, n}^{k} \sim$ $\mathcal{C N}\left(0, \sigma_{q, n}^{2}\right)$ is the channel coefficient between $\mathrm{BS}_{q}$ and user $U_{n} \in \mathcal{U}_{q}$ over channel $C_{q, k} \in \mathcal{C}_{q}$, and $\sigma_{q, n}^{2}=d_{q, n}^{-v}$ is the channel variance, with $v$ and $d_{q, n}$ being the path loss exponent and distance, respectively. Moreover, the instantaneous channel gain is defined as $g_{q, n}^{k} \triangleq\left|h_{q, n}^{k}\right|^{2}$. Thus, without loss of generality, let the channel gains between base station $\mathrm{BS}_{q}$ and the users within its vicinity over each channel $C_{q, k}$ be ordered as $g_{q, 1}^{k} \leq g_{q, 2}^{k} \leq \cdots \leq g_{q,\left|\mathcal{U}_{q}\right|}^{k}$.

\footnotetext{
${ }^{1}$ The SIC receiver complexity is $\mathcal{O}\left(\zeta_{q, k}^{3}\right), \forall C_{q, k} \in \mathcal{C}_{q}$, and $\forall B S_{q} \in \mathcal{B}$ [2].

${ }^{2}$ In other words, no frequency reuse is assumed in this work, and thus, inter-cell interference is not considered.

${ }^{3}$ Perfect CSI knowledge is assumed at the base stations.
}

In turn, the power allocation coefficients are ordered as $a_{q, 1}^{k} \geq a_{q, 2}^{k} \geq \cdots \geq a_{q,\left|\mathcal{U}_{q}\right|}^{k}$. Additionally, the total transmit power at each base station $\mathrm{BS}_{q}$ is set as $P_{\mathrm{BS}}, \forall \mathrm{BS}_{q} \in \mathcal{B}$. More importantly, let $P_{q, k}=P_{B S} /\left|\mathcal{C}_{q}\right| \triangleq P$ be the transmit power per allocated channel, $\forall C_{q, k} \in \mathcal{C}_{q}$ and $\forall \mathrm{BS}_{q} \in \mathcal{B}$, where $|\cdot|$ denotes the cardinality of the parameter set.

In this work, perfect SIC is assumed ${ }^{4}$, and thus, the signal-to-interference-plus-noise ratio (SINR) of user $U_{n} \in \mathcal{U}_{q}$ over channel $C_{q, k} \in \mathcal{C}_{q}$ is written as

$$
\gamma_{q, n}^{k}=\frac{\rho g_{q, n}^{k} a_{q, n}^{k}}{\rho g_{q, n}^{k} \bar{a}_{q, n}^{k}+1},
$$

with $\rho \triangleq P / N_{0}, \bar{a}_{q, n}^{k} \triangleq \sum_{m=n+1}^{\left|\mathcal{U}_{q}\right|} a_{q, m}^{k}$, and $\bar{a}_{q,\left|\mathcal{U}_{q}\right|}^{k}=0$.

Remark 1 The SINR function $\gamma_{q, n}^{k}, \forall n \neq\left|\mathcal{U}_{q}\right|$, is linearfractional (LF) function, with $\rho g_{q, n}^{k} a_{q, n}^{k}$ and $\rho g_{q, n}^{k} \bar{a}_{q, n}^{k}+1>$ 0 being linear functions in $a_{q, n}^{k}$ and $\bar{a}_{q, n}^{k}$, respectively [21]. However, the SNR function $\gamma_{q,\left|U_{q}\right|}^{k}$ is a linear function in $a_{q,\left|\mathcal{U}_{q}\right|}^{k}$

\section{Centralized joint user association, channel assignment, and power allocation}

In this work, the aim is to jointly associate users with base stations and perform channel assignment, along with proportional fairness SINR-based maximizing power allocation. Additionally, each user must satisfy a target minimum SINR $\gamma_{T_{n}}, \forall U_{n} \in \mathcal{U}_{q}$, and $\forall B S_{q} \in \mathcal{B}$ (i.e.,

\footnotetext{
${ }^{4}$ The case of imperfect SIC is beyond the scope of this work; however, practical solutions for mitigating SIC errors can be found in [20].
} 
QoS constraints). To this aim, define the binary decision variable $\mathcal{I}_{q, n}^{k}$ as

$\mathcal{I}_{q, n}^{k}=\left\{\begin{array}{l}1, \text { if channel } C_{q, k} \text { is assigned to user } U_{n} \in \mathcal{U}_{q}, \\ 0, \text { otherwise. }\end{array}\right.$

Therefore, the centralized joint user association, channel assignment, and power allocation (C-J-UA-CA-PA) problem is formulated as

\section{C-J-UA-CA-PA:}

$$
\begin{aligned}
& \max \prod_{U_{n} \in \mathcal{U}}\left(\sum_{B S_{q} \in \mathcal{B}} \sum_{C_{q, k} \in \mathcal{C}_{q}} \mathcal{I}_{q, n}^{k} \frac{\rho g_{q, n}^{k} a_{q, n}^{k}}{\rho g_{q, n}^{k} \bar{a}_{q, n}^{k}+1}\right. \\
& \left.+\left(1-\sum_{B S_{q} \in \mathcal{B}} \sum_{C_{q, k} \in \mathcal{C}_{q}} \mathcal{I}_{q, n}^{k}\right)\right) \\
& \text { s.t. } \mathcal{I}_{q, n}^{k}\left(\frac{\rho g_{q, n}^{k} a_{q, n}^{k}}{\rho g_{q, n}^{k} \bar{a}_{q, n}^{k}+1}-\gamma_{T_{n}}\right) \geq 0 \text {, } \\
& \forall C_{q, k} \in \mathcal{C}_{q}, \forall U_{n} \in \mathcal{U}_{q} \text { and } \forall \mathrm{BS}_{q} \in \mathcal{B} \text {, } \\
& \sum_{U_{n} \in \mathcal{U}_{q}} \sum_{C_{q, k} \in \mathcal{C}_{q}} \mathcal{I}_{q, n}^{k} \leq \xi_{q}, \quad \forall \mathrm{BS}_{q} \in \mathcal{B}, \\
& \sum_{U_{n} \in \mathcal{U}_{q}} \mathcal{I}_{q, n}^{k} \leq \zeta_{q, k}, \quad \forall C_{q, k} \in \mathcal{C}_{q} \text { and } \forall \mathrm{BS}_{q} \in \mathcal{B} \text {, } \\
& \sum_{\mathrm{BS}_{q} \in \mathcal{B}} \sum_{C_{q, k} \in \mathcal{C}_{q}} \mathcal{I}_{q, n}^{k} \leq 1, \quad \forall U_{n} \in \mathcal{U}, \\
& \sum_{U_{n} \in \mathcal{U}_{q}} a_{q, n}^{k} \leq 1, \quad \forall C_{q, k} \in \mathcal{C}_{q} \text { and } \forall \mathrm{BS}_{q} \in \mathcal{B}, \\
& a_{q, 1}^{k} \geq a_{q, 2}^{k} \geq \cdots \geq a_{q,\left|\mathcal{U}_{q}\right|}^{k}, \quad \forall C_{q, k} \in \mathcal{C}_{q}, \\
& \forall U_{n} \in \mathcal{U}_{q} \text { and } \forall \mathrm{BS}_{q} \in \mathcal{B} \text {, } \\
& 0 \leq a_{q, n}^{k} \leq \mathcal{I}_{q, n}^{k}, \quad \forall C_{q, k} \in \mathcal{C}_{q} \text {, } \\
& \forall U_{n} \in \mathcal{U}_{q} \text { and } \forall \mathrm{BS}_{q} \in \mathcal{B} \text {, } \\
& \mathcal{I}_{q, n}^{k} \in\{0,1\}, \quad \forall C_{q, k} \in \mathcal{C}_{q}, \forall U_{n} \in \mathcal{U}_{q} \text { and } \forall \mathrm{BS}_{q} \in \mathcal{B} \text {. }
\end{aligned}
$$

Constraint (3a) ensures that if a user $U_{n}$ is paired to a channel $C_{q, k}$, then it must satisfy the target minimum SINR $\gamma_{T_{n}}$, while constraint (3b) ensures that the total number of users paired to channels in any base station $\mathrm{BS}_{q}$ must not exceed $\xi_{q}$. Moreover, constraint (3c) ensures that the number of users paired to each channel $C_{q, k} \in \mathcal{C}_{q}$ is at most $\zeta_{q, k}$. Constraint (3d) ensures that a user is associated with at most one base station, while constraint (3e) ensures that the sum of power allocation coefficients of the assigned users over any channel does not exceed one.
Constraint (3f) ensures that the decoding order of the SIC is preserved. Constraint (3g) defines the range of values each power allocation coefficients can take. Particularly, if $\mathcal{I}_{q, n}^{k}=1$, then $0 \leq a_{q, n}^{k} \leq 1$; otherwise, $a_{q, n}^{k}=0$. Finally, the last constraint defines the range of values the binary decision variables can take.

Remark 2 The C-J-UA-CA-PA problem comprises an upper-bound total of $N \cdot \sum_{B S_{q} \in \mathcal{B}}\left|\mathcal{C}_{q}\right|$ continuous decision variables (i.e., $a_{q, n}^{k}$ ) and a similar number of binary decision variables (i.e., $\mathcal{I}_{q, n}^{k}$ ). In addition, it can be verified that there is an upper-bound total of $N+$ $Q+2\left(\sum_{B S_{q} \in \mathcal{B}}\left|\mathcal{C}_{q}\right|+\sum_{B S_{q} \in \mathcal{B}}\left|\mathcal{C}_{q}\right| \cdot\left|\mathcal{U}_{q}\right|\right)+\sum_{B S_{q} \in \mathcal{B}}\left|\mathcal{C}_{q}\right| \cdot$ $\left(\left|\mathcal{U}_{q}\right|-1\right)$ constraints.

Remark 3 Problem C-J-UA-CA-PA is a classified as a mixed-integer nonlinear programming problem (MINLP), which is NP-hard (i.e., extremely computationally expensive [22, 23]). Thus, it can only be solved via a global optimization package.

Based on Remark 3, problem C-J-UA-CA-PA can be decomposed in two sub-problems: (1) proportional fairness SINR-based maximizing power allocation and (2) stable matching-theoretic user association and channel assignment. Particularly, the aim is to optimally solve the SINR-maximizing power allocation per base station and over each channel, while accounting for inter-user interference and QoS constraints. This will be achieved via a low-complexity iterative solution procedure, which also determines the preferences of users over channels, and the preferences of base stations over users. After that, basestation association and channel assignment is performed via the stable matching algorithms.

\section{Proportional fairness SINR-based maximizing power allocation}

This section focuses on proportional fairness SINR-based maximizing (PR-SINR-MAX) power allocation, subject to target minimum SINR $\gamma_{T_{n}}$ per user $U_{n} \in \mathcal{U}_{q}$. Specifically, the objective function of base station $\mathrm{BS}_{q}$ over channel $C_{q, k} \in \mathcal{C}_{q}$ be given by

$$
\gamma_{q}^{k} \triangleq \prod_{U_{n} \in \mathcal{U}_{q}} \gamma_{q, n}^{k}=\prod_{U_{n} \in \mathcal{U}_{q}} \frac{\rho g_{q, n}^{k} a_{q, n}^{k}}{\rho g_{q, n}^{k} \bar{a}_{q, n}^{k}+1},
$$

which can be re-expressed as

$$
\gamma_{q}^{k}\left(\mathbf{a}_{q}^{k}, \mathcal{I}_{q}^{k}\right)=\prod_{U_{n} \in \mathcal{U}_{q}}\left(\mathcal{I}_{q, n}^{k} \frac{\rho g_{q, n}^{k} a_{q, n}^{k}}{\rho g_{q, n}^{k} \bar{a}_{q, n}^{k}+1}+\left(1-\mathcal{I}_{q, n}^{k}\right)\right),
$$


where $\mathbf{a}_{q}^{k}=\left[a_{q, 1}^{k}, a_{q, 2}^{k}, \ldots, a_{q,\left|\mathcal{U}_{q}\right|}^{k}\right]$ is the power allocation vector, while $\mathcal{I}_{q}^{k}=\left[\mathcal{I}_{q, 1}^{k}, \mathcal{I}_{q, 1}^{k}, \ldots, \mathcal{I}_{q,\left|\mathcal{U}_{q}\right|}^{k}\right]$ is the channel assignment vector. Since each channel $C_{q, k} \in$ $\mathcal{C}_{q}$ has a quota $\zeta_{q, k}$, then $\sum_{U_{n} \in \mathcal{U}_{q}} \mathcal{I}_{q, n}^{k} \leq \zeta_{q, k}$. The objective function in (5) stipulates that if user $U_{n}$ is assigned channel $C_{q, k}$ (i.e., $\mathcal{I}_{q, n}^{k}=1$ ), then its SINR function is maximized; otherwise, it is set to one. Therefore, the PR-SINR-MAX optimization problem can be formulated as

\section{PR-SINR-MAX:}

$$
\begin{array}{ll}
\max & \gamma_{q}^{k}\left(\mathbf{a}_{q}^{k}, \mathcal{I}_{q}^{k}\right)=\prod_{U_{n} \in \mathcal{U}_{q}}\left(\mathcal{I}_{q, n}^{k} \frac{\rho g_{q, n}^{k} a_{q, n}^{k}}{\rho g_{q, n}^{k} \bar{a}_{q, n}^{k}+1}+\left(1-\mathcal{I}_{q, n}^{k}\right)\right) \\
\text { s.t. } & \mathcal{I}_{q, n}^{k}\left(\frac{\rho g_{q, n}^{k} a_{q, n}^{k}}{\rho g_{q, n}^{k} \bar{a}_{q, n}^{k}+1}-\gamma_{T_{n}}\right) \geq 0, \quad \forall U_{n} \in \mathcal{U}_{q},
\end{array}
$$

$$
\begin{aligned}
& \sum_{U_{n} \in \mathcal{U}_{q}} a_{q, n}^{k} \leq 1, \\
& \sum_{U_{n} \in \mathcal{U}_{q}} \mathcal{I}_{q, n}^{k} \leq \zeta_{q, k}, \\
& a_{q, 1}^{k} \geq a_{q, 2}^{k} \geq \cdots \geq a_{q,\left|\mathcal{U}_{q}\right|}^{k}, \\
& 0 \leq a_{q, n}^{k} \leq \mathcal{I}_{q, n}^{k}, \quad \forall U_{n} \in \mathcal{U}_{q}, \\
& \mathcal{I}_{q, n}^{k} \in\{0,1\}, \quad \forall U_{n} \in \mathcal{U}_{q} .
\end{aligned}
$$

Constraint (6a) ensures that if a user is selected, then it must satisfy the target minimum SINR, while constraint (6b) ensures that the sum of power allocation coefficients of the selected users does not exceed one. Constraint (6c) ensures that the maximum number of users per channel $C_{q, k} \in \mathcal{C}_{q}$ does not exceed $\zeta_{q, k}$, while constraint (6d) maintains the SIC decoding order. Constraint (6e) ensures that if channel $C_{q, k}$ is assigned to user $U_{n}$ (i.e., $\mathcal{I}_{q, n}^{k}=1$ ), then the power allocation coefficient $a_{q, n}^{k}$ must not exceed one; otherwise, $a_{q, n}^{k}=0$ (since $\mathcal{I}_{q, n}^{k}=0$ ). The last constraint defines the range of values the binary decision variables take.

Remark 4 Problem PR-SINR-MAX is classified as a mixed-integer linear-fractional programming (MILFP) problem. More importantly, it is non-convex (and NP-hard [24]); and thus, it is still computationally expensive.

In turn, a simple solution procedure is devised to efficiently solve problem PR-SINR-MAX. To that end, let $\Omega_{q, k}=\left\{\Omega_{q, k, 1}, \ldots, \Omega_{q, k, \zeta_{q, k}}\right\}$ be the set of all combinations of assigning $u_{q}$ users in $\mathcal{U}_{q}$ (for $1 \leq u_{q} \leq$ $\zeta_{q, k}$ ) over each channel $C_{q, k} \in \mathcal{C}_{q}$, where $\Omega_{q, k, u_{q}}=$ $\left\{\omega_{q, k, 1}, \omega_{q, k, 2}, \ldots, \omega_{q, k, \omega_{u_{q}}}\right\}$, and

$$
\varpi_{u_{q}} \triangleq\left(\begin{array}{c}
\left|\mathcal{U}_{q}\right| \\
u_{q}
\end{array}\right)=\frac{\left|\mathcal{U}_{q}\right| !}{\left(\left|\mathcal{U}_{q}\right|-u_{q}\right) ! u_{q} !},
$$

with $\varpi_{u_{q}}=\left|\Omega_{q, k, u_{q}}\right|$. Each combination is defined as $\omega_{q, k, 5}=\left[\mathcal{J}_{q, 5,1}^{k}, \mathcal{J}_{q, 5,2}^{k}, \ldots, \mathcal{J}_{\left.q, 5, \mid \mathcal{U}_{q}\right]}^{k}\right]$, for $\varsigma=1,2, \ldots, \varpi_{u_{q}}$. Specifically, $\mathcal{J}_{q, 5, n}^{k}$ is defined as

$\mathcal{J}_{q, 5, n}^{k}=\left\{\begin{array}{l}1, \text { if } U_{n} \in \mathcal{U}_{q} \text { is assigned } C_{q, k} \in \mathcal{C}_{q} \text { in } \omega_{q, k, 5}, \\ 0, \text { otherwise. }\end{array}\right.$

For example, if $\left|\mathcal{U}_{q}\right|=3$ and $\zeta_{q, k}=2$, then $\Omega_{q, k}=$ $\left\{\Omega_{q, k, 1}, \Omega_{q, k, 2}\right\}$, where $\Omega_{q, k, 1}$ includes the combinations $[1,0,0],[0,1,0]$, and $[0,0,1]$, while $\Omega_{q, k, 2}$ contains the combinations $[1,1,0],[1,0,1]$, and $[0,1,1]$. In other words, in the first set of combinations $\Omega_{q, k, 1}$, only one user is assumed to be assigned to channel $C_{q, k}$, while in $\Omega_{q, k, 2}$, different combinations of two users are assigned to that channel.

Remark 5 The total number of all possible user combinations over each channel $C_{q, k} \in \mathcal{C}_{q}, \forall \mathrm{BS}_{q} \in \mathcal{B}$ is obtained as

$$
\Sigma\left(\mathcal{U}_{q}, \zeta_{q, k}\right)=\sum_{u_{q}=1}^{\zeta_{q, k}} \frac{\left|\mathcal{U}_{q}\right| !}{\left(\left|\mathcal{U}_{q}\right|-u_{q}\right) ! u_{q} !} .
$$

For notational convenience, let $\mathcal{U}_{q, k, \varsigma} \subseteq \mathcal{U}_{q}$ be the subset of users in $\mathcal{U}_{q}$ with $\mathcal{J}_{q, 5, n}^{k}=1$ in $\omega_{q, k, 5} \in \Omega_{q, k, u_{q}}$. Thus, the SINR of each user $U_{n} \in \mathcal{U}_{q, k, 5}$ is expressed as

$$
\gamma_{q, n, 5}^{k}=\frac{\rho g_{q, n, \zeta}^{k} a_{q, n, \zeta}^{k}}{\rho g_{q, n, \zeta}^{k} \bar{a}_{q, n, 5}^{k}+1},
$$

It can be seen that for each possible combination $\omega_{q, k, \varsigma} \in$ $\Omega_{q, k, u_{q}}$, each user $U_{n} \in \mathcal{U}_{q, k, 5}$ will have a different SINR value, which depends on the other users sharing the same channel in that combination.

Remark 6 The SINR function of each user is one with peer effects (also known as negative network externality), which is due to the fact that each user's SINR $\gamma_{q, n, 5}^{k}$ is influenced by the allocated power to all the other users utilizing the same channel.

Remark 7 For $u_{q}=1$, only one user is assigned to each channel $C_{q, k} \in \mathcal{C}_{q}$, in any combination $\omega_{q, k, 5}$ in $\Omega_{q, k, 1}, \forall \zeta=1,2, \ldots,\left|\mathcal{U}_{q}\right|$. Consequently, the power allocation coefficient for each user $U_{n}$ with $\mathcal{J}_{q, 5, n}^{k}=1$ 
in $\omega_{q, k, 5}$ is $a_{q, n, 5}^{k}=1$, whereas $\bar{a}_{q, n, 5}^{k}=0$, and hence, $\gamma_{q, n, 5}^{k}=\rho g_{q, n, 5}^{k}$

Now, for each possible combination $\omega_{q, k, 5} \in \Omega_{q, k, u_{q}}$, for $2 \leq u_{q} \leq \zeta_{q, k}$, problem PR-SINR-MAX can be re-written as

\section{$\underline{\text { PR-SINR-MAX }\left(\omega_{q, k, 5}\right):}$}

$\max \quad \gamma_{q, \zeta}^{k}\left(\mathbf{a}_{q, \zeta}^{k}, \omega_{q, k, \zeta}\right)=\prod U_{U_{n} \in \mathcal{U}_{q, k, 5}} \frac{\rho g_{q, n, 5}^{k} a_{q, n, 5}^{k}}{\rho g_{q, n, 5}^{k} \bar{a}_{q, n, 5}^{k}+1}$

s.t. $\rho g_{q, n, \zeta}^{k} a_{q, n, \varsigma}^{k} \geq \gamma_{T_{n}}\left(\rho g_{q, n, \zeta}^{k} \bar{a}_{q, n, \varsigma}^{k}+1\right), \quad \forall U_{n} \in \mathcal{U}_{q, k, \zeta}$,

$$
\begin{aligned}
& \sum_{U_{n} \in \mathcal{U}_{q, k, \zeta}} a_{q, n, \zeta}^{k} \leq 1, \\
& a_{q, 1,5}^{k} \geq a_{q, 2, \varsigma}^{k} \geq \cdots \geq a_{q,\left|\mathcal{U}_{q, k, \zeta}\right|, 5}^{k} \\
& 0 \leq a_{q, n, 5}^{k} \leq 1, \quad \forall U_{n} \in \mathcal{U}_{q, k, \zeta},
\end{aligned}
$$

which can be verified to be non-convex, although all the constraints are linear [25]. By utilizing the fact that the $\ln (\cdot)$ function is concave and strictly monotonically increasing, then the objective function of problem $P R$ $\operatorname{SINR}-M A X\left(\omega_{q, k, 5}\right)$ can be re-expressed as

$$
\ln \gamma_{q}^{k}\left(\mathbf{a}_{q, \zeta}^{k}, \omega_{q, k, \zeta}\right)=f\left(\mathbf{a}_{q, \zeta}^{k}\right)-g\left(\mathbf{a}_{q, \zeta}^{k}\right),
$$

with $f\left(\mathbf{a}_{q, \zeta}^{k}\right)$ being defined as

$$
f\left(\mathbf{a}_{q, \zeta}^{k}\right)=\sum_{U_{n} \in \mathcal{U}_{q, k, \zeta}} \ln \left(\rho g_{q, n, \varsigma}^{k} a_{q, n, \varsigma}^{k}\right),
$$

while

$$
g\left(\mathbf{a}_{q, \zeta}^{k}\right)=\sum_{U_{n} \in \mathcal{U}_{q, k, \zeta}} \ln \left(\rho g_{q, n, \zeta}^{k} \bar{a}_{q, n, \zeta}^{k}+1\right) .
$$

It is noteworthy that $f\left(\mathbf{a}_{q, 5}^{k}\right)$ and $g\left(\mathbf{a}_{q, 5}^{k}\right)$ are concave functions, which are also twice continuously differentiable in $a_{q, n, 5}^{k}$ and $\bar{a}_{q, n, 5}^{k}$, respectively. Hence, $\ln \gamma_{q, \zeta}^{k}\left(\mathbf{a}_{q, \zeta}^{k}, \omega_{q, k, \zeta}\right)$ is a difference convex function [26, 27]. As a result, problem PR-SINR-MAX $\left(\omega_{q, k, 5}\right)$ can be reformulated as $[28,29]$

$$
\begin{aligned}
& \frac{\text { PR-SINR-MAX }\left(\omega_{q, k, 5}\right):}{\min \quad \mu-g\left(\mathbf{a}_{q, \zeta}^{k}\right)} \\
& \text { s.t. } \quad f\left(\mathbf{a}_{q, \zeta}^{k}\right) \leq \mu,
\end{aligned}
$$

$$
\text { Constraints (11a)-(11d), }
$$

$$
\mu \geq 0,
$$

with $\mu$ being an auxiliary variable. It can be easily verified that the objective function and the first constraint are concave functions in $a_{q, n, \zeta}^{k}, \forall U_{n} \in \mathcal{U}_{q, k, \zeta}$, whereas the remaining constraints are linear.

Remark 8 R-PR-SINR-MAX $\left(\omega_{q, k, 5}\right)$ is a concave minimization problem [30] and thus is solved efficiently with minimal complexity via any standard convex optimization package [31].

Remark 9 The optimal solution of problem R-PR-SINR$\operatorname{MAX}\left(\omega_{q, k, 5}\right)$ is also the optimal solution of the original problem PR-SINR-MAX $\left(\omega_{q, k, 5}\right)$ [30].

The following solution procedure determines the resulting SINR per user $U_{n}$ for each channel $C_{q, k}$ of each base station $\mathrm{BS}_{q}$. The goal is to iterate over all possible user combinations in $\Omega_{q, k}$. Specifically, the solution procedure starts by considering single-user combinations (i.e., when $u_{q}=1$ and for each $\omega_{q, k, 5} \in \Omega_{q, k, 1}$ ) over each channel $C_{q, k} \in \mathcal{C}_{q}$ and determines whether the target minimum SINR $\gamma_{T_{n}}$ is satisfied by calculating $\gamma_{q, n, 5}^{k}, \forall U_{n} \in \mathcal{U}_{q, k, 5}$. If $\gamma_{q, n, 5}^{k}<\gamma_{T_{n}}$ for any $\forall U_{n} \in$ $\mathcal{U}_{q, k, \zeta}$, then set $\gamma_{q, n, 5}^{k}=0$; otherwise, keep the value of $\gamma_{q, n, \varsigma}^{k}$ for later use to determine the preference lists. After that, the solution procedure considers user combinations of sizes $2 \leq u_{q} \leq \zeta_{q, k}$, and solves problem R-PR-SINR-MAX $\left(\omega_{q, k, 5}\right)$ for each combination, such that the optimal SINR of each user in each combination over each channel is evaluated to determine whether the target minimum SINR is satisfied, as stated earlier. Upon completion of the solution procedure, each user $U_{n} \in \mathcal{U}_{q}$ calculates

$$
\chi_{q, n}^{k} \triangleq \sum_{u_{q}=1}^{\zeta_{q, k}} \sum_{\varsigma=1}^{\varpi_{u_{q}}} \mathcal{J}_{q, \zeta, n}^{k} \cdot \gamma_{q, n, \zeta}^{k}
$$

for each channel $C_{q, k} \in \mathcal{C}_{q}$, which is considered as a weight of how valuable that channel is to that user. Contrarily, each base station $\mathrm{BS}_{q} \in \mathcal{B}$ calculates

$$
\psi_{q, n} \triangleq \sum_{C_{q, k} \in \mathcal{C}_{q}} \sum_{u_{q}=1}^{\zeta_{q, k}} \sum_{\varsigma=1}^{\varpi_{u_{q}}} \mathcal{J}_{q, \zeta, n}^{k} \cdot \gamma_{q, n, \zeta}^{k}
$$

for each user $U_{n} \in \mathcal{U}_{q}$, which is used as a weight for how valuable that user is to that base station.

The proposed solution procedure for proportional fairness SINR-based maximization (SP-PF-SINR-MAX) is outlined in Algorithm 1, which can be performed locally at each base station. 


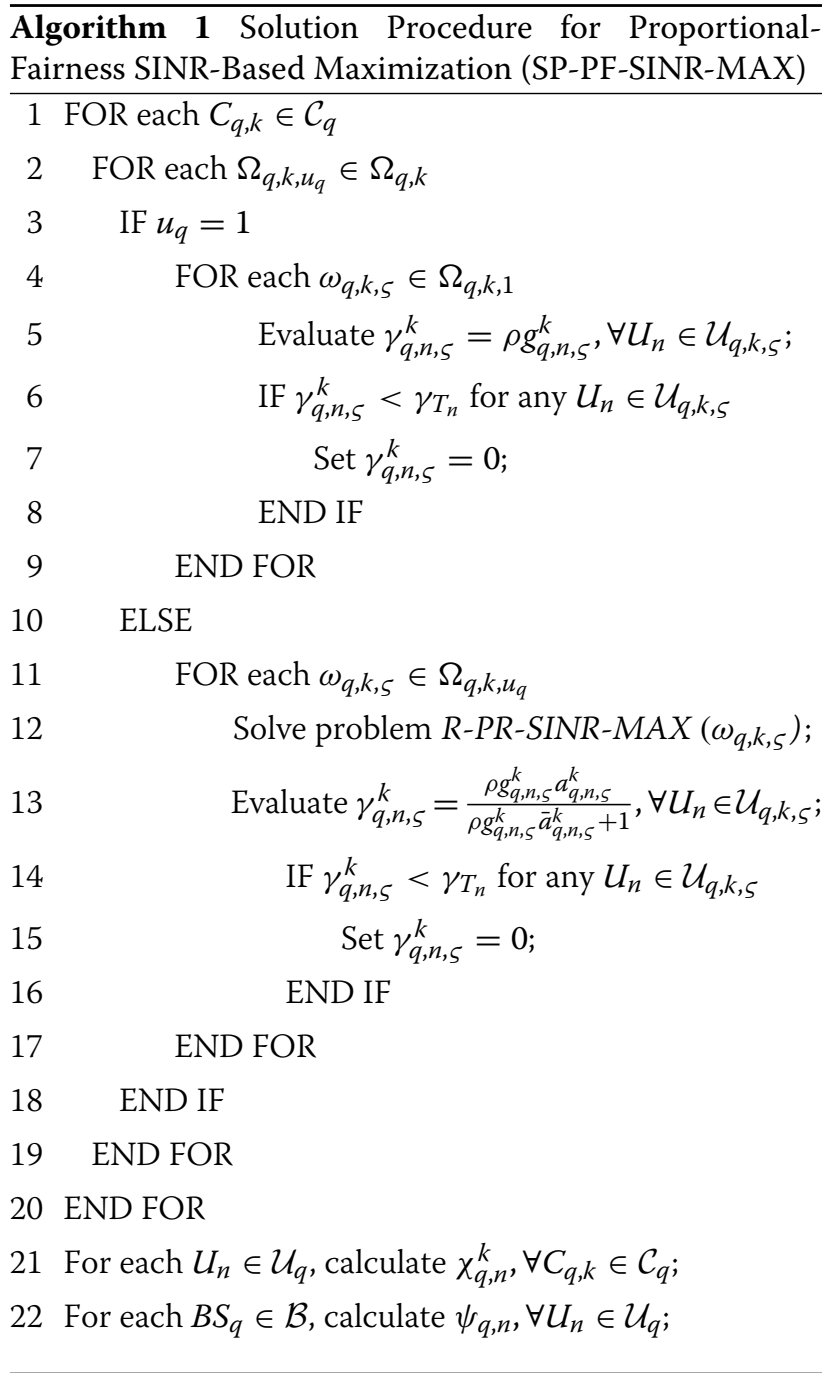

Remark 10 For the case when $u_{q}=1$ (i.e., only combinations $\omega_{q, k, \zeta} \in \Omega_{q, k, 1}$, for $\left.\zeta=1,2, \ldots, \varpi_{1}\right)$ in which only a single user is considered over each channel $C_{q, k} \in \mathcal{C}_{q}$ (i.e. $\mathcal{J}_{q, 1, n}^{k}=1$ ), if a user $U_{n} \in \mathcal{U}_{q, k, 5}$ cannot satisfy the target minimum SINR $\gamma_{T_{n}}$, then it cannot satisfy it when a greater number of users are considered (i.e., when $2 \leq$ $u_{q} \leq \zeta_{q, k}$ ) over that channel (due to negative network externality). Hence, $\chi_{q, n}^{k}=0$ for user $U_{n} \in \mathcal{U}_{q}$ over channel $C_{q, k} \in \mathcal{C}_{q}$

Remark 11 The greater the value of $\chi_{q, n}^{k}$ is, the more preferred channel $C_{q, k} \in \mathcal{C}_{q}$ is to user $U_{n} \in \mathcal{U}_{q}$. In a similar manner, the greater the value of $\psi_{q, n}$ is, the more preferred user $U_{n} \in \mathcal{U}_{q}$ is to base station $B S_{q} \in \mathcal{B}$.

Remark 12 Iffor any user $U_{n} \in \mathcal{U}_{q}$, the value of $\chi_{q, n}^{k}=0$, then that channel is considered unacceptable to that user.
In a similar manner, if, for a base station $B S_{q} \in \mathcal{B}, \psi_{q, n}=$ 0 , then user $U_{n}$ is deemed unacceptable.

Remark 13 At the outset, the proposed solution procedure may seem to be excessively complex. However, for each base station $B S_{q} \in \mathcal{B}$, there is a total of $\left|\mathcal{C}_{q}\right| \cdot \Sigma\left(\mathcal{U}_{q}, \zeta_{q, k}\right)$ iterations, and a convex optimization problem is efficiently solved in only $\left|\mathcal{C}_{q}\right| \cdot \Sigma\left(\mathcal{U}_{q}, \zeta_{q, k}\right)-\left|\mathcal{U}_{q}\right|$ iterations and thus is guaranteed to converge $e^{5}$.

It should be noted that in multi-cell NOMA networks, a small number of users are to be multiplexed into a channel, which is mainly to minimize inter-user interference, reduce SIC hardware complexity and error propagation, and leverage capacity gains [7], while satisfying the target minimum SINR constraint per user (i.e., $\zeta_{q, k}$ must be kept reasonably small, $\forall C_{q, k} \in \mathcal{C}_{q}$ ). Lastly, the proposed solution procedure eliminates the need for swap operations, which have been utilized in [17-19]. Specifically, in the aforementioned references, a swap operation is utilized in an iterative manner to ensure stability after power allocation. However, in the solution procedure, the optimal power allocation per user over each channel is already determined, which is then used to determine the preference lists in the proposed matching algorithms (discussed in the following section). Consequently, the stable matching solutions resulting from the proposed stable matching algorithms will already have obtained the optimal power allocation for the users sharing each channel within each base station.

\section{Stable matching algorithms}

In this section, the stable matching algorithms based on the SPA problem are devised.

\subsection{Description}

The classical SPA problem involves a set of students (users), projects (channels), and lecturers (base stations). Each project is offered by a specific lecturer, and each lecturer has quotas (i.e., a maximum number of students per project and per lecturer, respectively). Moreover, students have preferences over the projects that are acceptable (i.e., in which they would like to be involved), while each lecturer has preferences over the acceptable students ${ }^{6}$. Typically, a project is assigned to at most one student, while in some other cases, a project may be undertaken by more than one student to work on. More importantly, there should be a number of projects that coincides with the number of potential students, and each lecturer is responsible for offering a range of projects, which are not

\footnotetext{
${ }^{5}$ Each convex optimization problem is solved efficiently within polynomial-time complexity [31].

${ }^{6}$ The preferences are ordered in a descending order, from most preferred to the least.
} 
necessarily all taken up. By utilizing the students' and lecturers' preferences, the goal is to find a stable matching that pairs students to projects offered by each lecturer, while satisfying the quotas $[14,15]$. In an analogous manner, the goal is to assign users to channels available at each base station, such that a stable matching solution is obtained, while satisfying the quotas of the channels and base stations.

To that end, a few definitions must first be given.

\subsection{Definitions}

Definition 1 (Acceptability) A channel $C_{q, k} \in \mathcal{C}_{q}, \forall B S_{q}$ $\in \mathcal{B}$, is said to be acceptable to user $U_{n} \in \mathcal{U}_{q}$ if $\chi_{q, n}^{k}>$ 0 . Thus, let $\mathcal{A}_{U_{n}}$ be the list of acceptable channels by user $U_{n}$. In a similar fashion, $\mathcal{A}_{B S_{q}}$ is the list of acceptable users $U_{n} \in \mathcal{U}_{q}$ to base station $B S_{q}$ (i.e., for which $\psi_{q, n}>0$ ).

Definition 2 (Assignment) An assignment $\mathcal{M}$ is defined as a subset of $\mathcal{U} \times \mathcal{C}$, such that:

(a) $\left(U_{n}, C_{q, k}\right) \in \mathcal{M}$ (i.e., user $U_{n}$ finds channel $C_{q, k}$ acceptable).

(b) A user $U_{n}$ is assigned at most one channel (i.e., $\mid\left\{\left(U_{n}, C_{q, k}\right) \in \mathcal{M}\right.$ for $\left.\left.C_{q, k} \in \mathcal{A}_{U_{n}}\right\} \mid \leq 1\right)$.

In turn, if $\left(U_{n}, C_{q, k}\right) \in \mathcal{M}$, then user $U_{n}$ is considered to be assigned to channel $C_{q, k}$, and vice versa (i.e., $C_{q, k}$ is assigned to $\left.U_{n}\right)$. Also, let $\mathcal{M}\left(U_{n}\right)=C_{q, k}$ indicate that channel $C_{q, k}$ is assigned to user $U_{n}$ in $\mathcal{M}$, whereas $\mathcal{M}\left(C_{q, k}\right)=U_{n}$ indicates that user $U_{n}$ is assigned to channel $C_{q, k}$.

Definition 3 (Preference) If user $U_{n}$ prefers channel $C_{q, k}$ to $C_{w, l}$ (i.e., $\chi_{q, n}^{k}>\chi_{w, n}^{l}$ for $\left.k \neq l\right)^{7}$, then $C_{q, k} \succ_{U_{n}} C_{w, l}$. In a similar fashion, if base station $B S_{q}$ prefers user $U_{n}$ to $U_{m}$ (i.e., $\psi_{q, n}>\psi_{q, m}$ for $n \neq m$ ), then $U_{n} \succ_{B S_{q}} U_{m}$.

Definition 4 (Preference list) Let $\mathbb{P}_{U_{n}}=\left\{C_{q, k}^{(1)}, \ldots\right.$, $\left.C_{w, l}^{\left(\left|\mathcal{A}_{U_{n}}\right|\right)}\right\}$ denote the preference list of user $U_{n}$, where $C_{q, k}^{(1)}$ $\left(C_{w, l}^{\left(\left|\mathcal{A}_{U_{n}}\right|\right)}\right)$ refers to the most (least) preferred channel to $U_{n}$. Similarly, $\mathbb{P}_{B S_{q}}=\left\{U_{n}^{(1)}, \ldots, U_{m}^{\left(\left|\mathcal{A}_{B S_{q}}\right|\right)}\right\}$ denotes the preference list of base station $B S_{q}$, where $U_{n}^{(1)}\left(U_{m}^{\left(\left|\mathcal{A}_{B S_{q}}\right|\right)}\right)$ refers to the most (least) preferred user to $B S_{q}$.

Definition 5 (Projected preference list) Let $\overline{\mathbb{P}}_{B S_{q}}^{k}, \forall B S_{q} \in \mathcal{B}$, be the projected preference list of base station $B S_{q}$, which is obtained from $\mathbb{P}_{B S_{q}}$ by eliminating the users who find channel $C_{q, k} \in \mathcal{C}_{q}$ unacceptable.

${ }^{7}$ It is noteworthy that $C_{w, l}$ refers to a channel different from $C_{q, k}$ under a possibly different base station $\mathrm{BS}_{w}$.
Definition 6 (Subscription) $A$ channel $C_{q, k} \in \mathcal{C}_{q}$ is said to be under-subscribed, full, or over-subscribed if $\left|\mathcal{M}\left(C_{q, k}\right)\right|$ is less than, equal to, or greater than $\zeta_{q, k}$, respectively. In a similar manner, for any base station $B S_{q} \in \mathcal{B}$ under assignment $\mathcal{M}$ (i.e., $\mathcal{M}\left(B S_{q}\right)$ ), $B S_{q}$ is considered to be under-subscribed, full, or oversubscribed if $\left|\mathcal{M}\left(B S_{q}\right)\right|$ is less, equal to, or greater than $\xi_{q}$, respectively.

Definition 7 (Matching) A matching $\mathcal{M}$ is an assignment, such that [14]:

(a) For each channel $C_{q, k} \in \mathcal{C}_{q},\left|\mathcal{M}\left(C_{q, k}\right)\right| \leq \zeta_{q, k}$.

(b) For each base station $B S_{q} \in \mathcal{B},\left|\mathcal{M}\left(B S_{q}\right)\right| \leq \xi_{q}$.

In other words, under matching $\mathcal{M}$, no channel $C_{q, k} \in$ $\mathcal{C}_{q}$ is assigned to more than $\zeta_{q, k}$ users and that each base station $B S_{q}$ is assigned at most $\xi_{q}$ users.

Definition 8 (Blocking) The pair $\left(U_{n}, C_{q, k}\right) \in(\mathcal{U} \times \mathcal{C}) \backslash$ $\mathcal{M}$ is said to block a matching $\mathcal{M}$ if [15]:

(a) $C_{q, k} \in \mathcal{A}_{U_{n}}$ (i.e., user $U_{n}$ finds $C_{q, k}$ acceptable).

(b) Either $U_{n}$ is unassigned in $\mathcal{M}$ or $U_{n}$ prefers $C_{w, l}$ to $\mathcal{M}\left(U_{n}\right)$.

(c) Either

(c1) $C_{q, k}$ is under-subscribed, and $B S_{q}$ is under-subscribed, or

(c2) $C_{q, k}$ is under-subscribed, $B S_{q}$ is full, and either $U_{n} \in \mathcal{M}\left(B S_{q}\right)$ or $B S_{q}$ prefers $U_{n}$ to the worst user in $\mathcal{M}\left(B S_{q}\right)$, or

(c3) $C_{q, k}$ is full and $B S_{q}$ prefers $U_{n}$ to the worst user in $\mathcal{M}\left(C_{q, k}\right)$.

Accordingly, a (student, project) pair satisfying the above conditions should not be included in the matching solution, as such matching would not be stable.

Definition 9 (Stable matching) A matching $\mathcal{M}$ is considered stable if $\mathcal{M}$ contains no blocking pairs.

\subsection{Algorithmic design}

In this subsection, the user-oriented stable matching (U-SM) and base station-oriented stable matching (BSSM) algorithms are described [15].

\subsubsection{User-oriented stable matching}

Initially, all users are assumed to be free, and all channels and base stations are completely unsubscribed. The $U$-SM algorithm is based on a sequence of proposals by each user to the acceptable channels in its preference list. Such proposals lead to provisional assignments between users, channels, and base stations, which may be modified 
during the execution of the algorithm. That is, entries from the preference lists of users and the preference lists of base stations may be eliminated. To be more specific, deleting a pair $\left(U_{n}, C_{q, k}\right)$ corresponds to eliminating channel $C_{q, k}$ from user $U_{n}$ 's preference list $\mathbb{P}_{U_{n}}$ and also removing $U_{n}$ from the projected list $\overline{\mathbb{P}}_{\mathrm{BS}_{q}}^{k}$ of base station $\mathrm{BS}_{q}$, which offers channel $C_{q, k}$. The algorithm iterates as long as there is some user with a non-empty preference list and who is still unpaired to any channel under any base station. If some channel $C_{q, k}$ is over-subscribed, then its base station rejects the worst user $U_{m}$ assigned to that channel (i.e., the pair $\left(U_{m}, C_{q, k}\right)$ is deleted). Also, if some base station $\mathrm{BS}_{q}$ is over-subscribed, then it rejects its worst assigned user $U_{m}$ and deletes the pair $\left(U_{m}, C_{q, l}\right)$, where $C_{q, l}$ is the channel previously assigned to user $U_{m}$. On the other hand, if a channel $C_{q, k}$ is full, then for the worst user $U_{m}$ paired to the channel (as per $\overline{\mathbb{P}}_{\mathrm{BS}_{q}}^{k}$ ), delete the pair of each successive user $U_{p}$ with that channel (i.e., $\left.\left(U_{p}, C_{q, k}\right)\right)$. Lastly, if some base station $\mathrm{BS}_{q}$ is full, then for the worst user $U_{m}$ associated with $\mathrm{BS}_{q}$, delete the pair $\left(U_{p}, C_{q, l}\right)$ of each successive user $U_{p}$ (in $\mathbb{P}_{\mathrm{BS}_{q}}$ ) with each channel $C_{q, l}$ that $U_{p}$ finds acceptable by base station $\mathrm{BS}_{q}$. The $U-S M$ algorithm is outlined in Algorithm 2.

\subsubsection{Base station-oriented stable matching}

In a similar manner to the $U-S M$ algorithm, all users are initially assumed to be free, and all channels and base stations are totally unsubscribed. Now, the BS-SM algorithm iterates over each base station $\mathrm{BS}_{q}$ that is under-subscribed and offers a channel $C_{q, k}$ to a user $U_{n}$, which is the first on $\mathrm{BS}_{q}$ 's preference list $\mathbb{P}_{\mathrm{BS}_{q}}$, and $C_{q, k}$ must be the first under-subscribed channel on $U_{n}$ 's preference list $\mathbb{P}_{U_{n}}$, such that $U_{n} \in \overline{\mathbb{P}}_{\mathrm{BS}_{q}}^{k}$. After breaking any existing assignment of user $U_{n}$, then it is provisionally assigned to channel $C_{q, k}$ under base station $\mathrm{BS}_{q}$. In turn, any pair $\left(U_{n}, C_{w, l}\right)$ to which user $U_{n}$ prefers $C_{q, k}$ to $C_{w, l}$ is deleted, and hence, $C_{w, l}$ is removed from $U_{n}$ 's preference list, and $U_{n}$ is eliminated from the projected list $\overline{\mathbb{P}}_{\mathrm{BS}_{w}}^{l}$ of base station $\mathrm{BS}_{w}$ offering channel $C_{w, l}$. This process is repeated until convergence, as given in Algorithm 3.

Remark 14 Backhaul links can be utilized to efficiently execute the U-SM and BS-SM algorithms among the base stations with minimal overhead [32,33] and without the need for a centralized controller.

\subsection{Properties}

In the following subsections, the properties of the $U-S M$ and BS-SM algorithms are discussed. To be concised, the properties of the $U$-SM algorithm are discussed, which are also applicable to the $B S-S M$ algorithm.

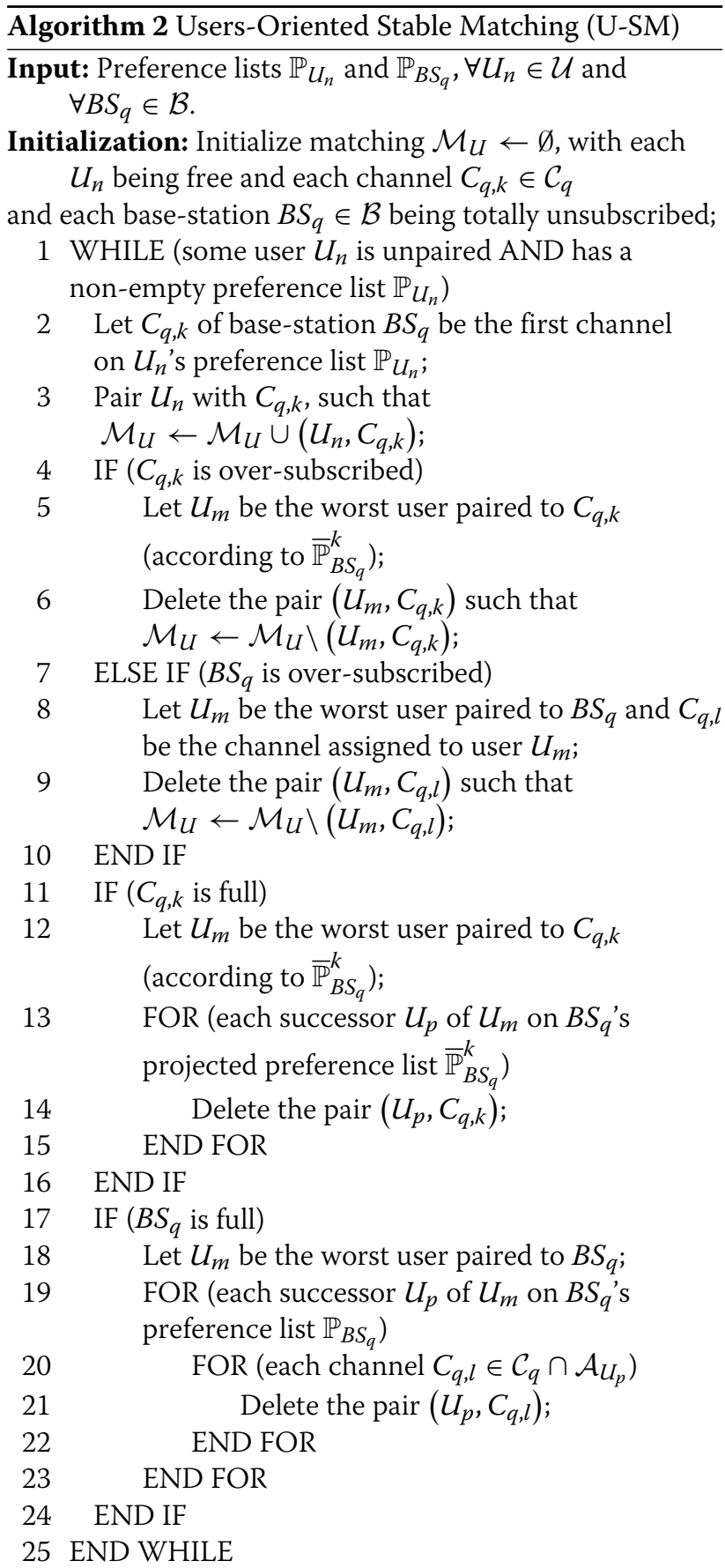

Output: Stable matching $\mathcal{M}_{U}$.

\subsubsection{Convergence to a stable matching solution \\ Lemma 1 The U-SM algorithm converges in a finite number of iterations to a stable matching solution.}

Proof In the $U-S M$ algorithm, a free user applies to the first channel on its preference list in each iteration. In particular, no user can apply to the same channel more 


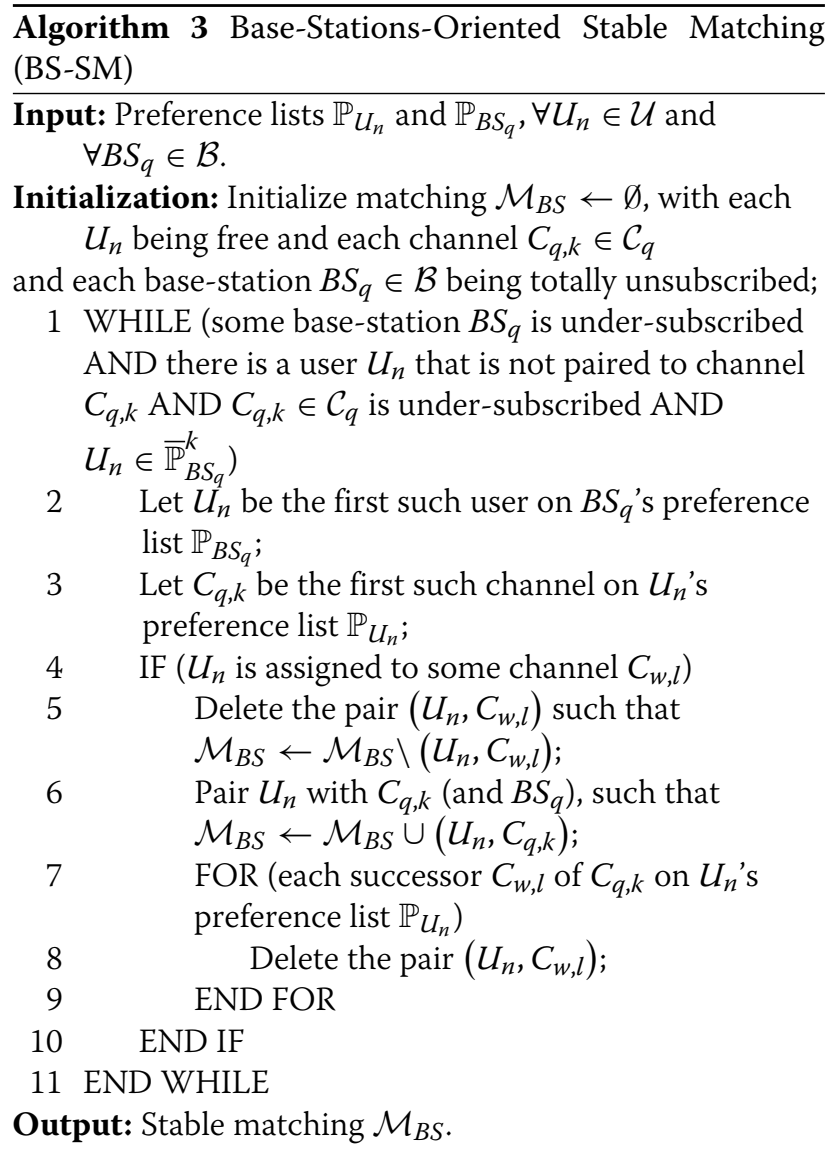

than once (i.e., each user-channel pair can occur at most once). This can be verified from the fact that once a pair $\left(U_{m}, C_{q, k}\right)$ is deleted, then user $U_{m}$ is freed and cannot apply to that channel again. This also implies that no pair deleted during the execution of the $U-S M$ algorithm can block the matching under construction [15]. If this had not been the case (i.e., the pair $\left(U_{m}, C_{q, k}\right)$ is not deleted), then user $U_{m}$ must be assigned to some channel $\mathcal{M}_{U}\left(U_{m}\right) \neq$ $C_{q, k}$; otherwise, user $U_{m}$ remains free with a non-empty preference list containing channel $C_{q, k}$ (i.e., a contradiction). Therefore, the pair $\left(U_{m}, C_{q, k}\right)$ must be deleted, since if $U_{m}$ prefers $C_{q, k}$ to $\mathcal{M}_{U}\left(U_{m}\right)$, then $\left(U_{m}, C_{q, k}\right)$ must block $\mathcal{M}_{U}$. More importantly, the $U-S M$ algorithm never deletes a stable pair during its execution [15]. Lastly, since the lengths of the user preferences lists are bounded, then the total number of iterations is also bounded. Hence, the $U-S M$ algorithm converges in a finite number of iterations to a stable matching solution.

\subsubsection{Complexity}

Lemma 2 The U-SM algorithm converges with polynomial-time complexity of $\mathcal{O}(|\mathcal{U}| \cdot|\mathcal{C}|)$, where $|\mathcal{U}|$ and $|\mathcal{C}|$ are the total number of users and channels, respectively [15].
Proof It is straightforward to verify that in the worst-case scenario of the $U$-SM algorithm, each free user $U_{n} \in$ $\mathcal{U}$-with a non-empty preference list-applies to at least one channel out of the possible $|\mathcal{C}|$ channels. Moreover, throughout the execution of the algorithm, some userchannel pairs may be deleted, and their corresponding entries are deleted from the preference lists of users and from the projected preference lists of base stations. Hence, the overall worst-case complexity of the $U-S M$ algorithm is $\mathcal{O}(|\mathcal{U}| \cdot|\mathcal{C}|)$.

\subsubsection{Optimality}

Lemma 3 The stable matching resulting from the U-SM algorithm is optimal with respect to each assigned user (i.e., user-optimal stable matching).

Proof Since each user is assigned to its first preferred channel on its reduced preference list, and no stable pair is deleted during the execution of the $U-S M$ algorithm [15], then each user is simultaneously assigned to the best channel it can get in any stable matching. In addition, and due to the proposed solution procedure SP-PF-SINR$M A X$, each user is not only assigned to its best channel, but also is allocated power optimally over its assigned channel.

By similar arguments, the BS-SM algorithm can straightforwardly be verified to converge to a stable matching solution within a finite number of iterations, and with polynomial-time complexity of $\mathcal{O}(|\mathcal{U}| \cdot|\mathcal{C}|)[15]$. It should be noted that the complexity of both the $U$ $S M$ and $B S-S M$ algorithms may be much lower than $\mathcal{O}(|\mathcal{U}| \cdot|\mathcal{C}|)$, which is due to the fact that each user $U_{n} \in$ $\mathcal{U}_{q}$ only has preferences over the channels within its cell (i.e., $\mathcal{C}_{q}$ ). In other words, it is only the users within the overlapping region of all cells that may have preferences over all channels. Last-but-not-least, the stable matching resulting from the $B S-S M$ algorithm is simultaneously optimal with respect to each base station. That is, each base station is associated with the best set of users it can get.

Despite the optimality of the proportional fairness SINR-based maximizing power allocation (and hence the solution procedure SP-PF-SINR-MAX), and also the optimality of the proposed stable matching algorithms (i.e., $U-S M$ and $B S-S M)$, the resulting base-station association, channel assignment, and power allocation solutions are not necessarily global optimal. This is due to the following two reasons. Firstly, decomposing problem C-J-UA-CA$P A$ into two sub-problems does not necessarily guarantee the global optimal solution. Secondly, enforcing stability in terms of the user association and channel assignment via the proposed stable matching algorithms may lead to a sub-optimal solution. Nevertheless, our algorithmic 


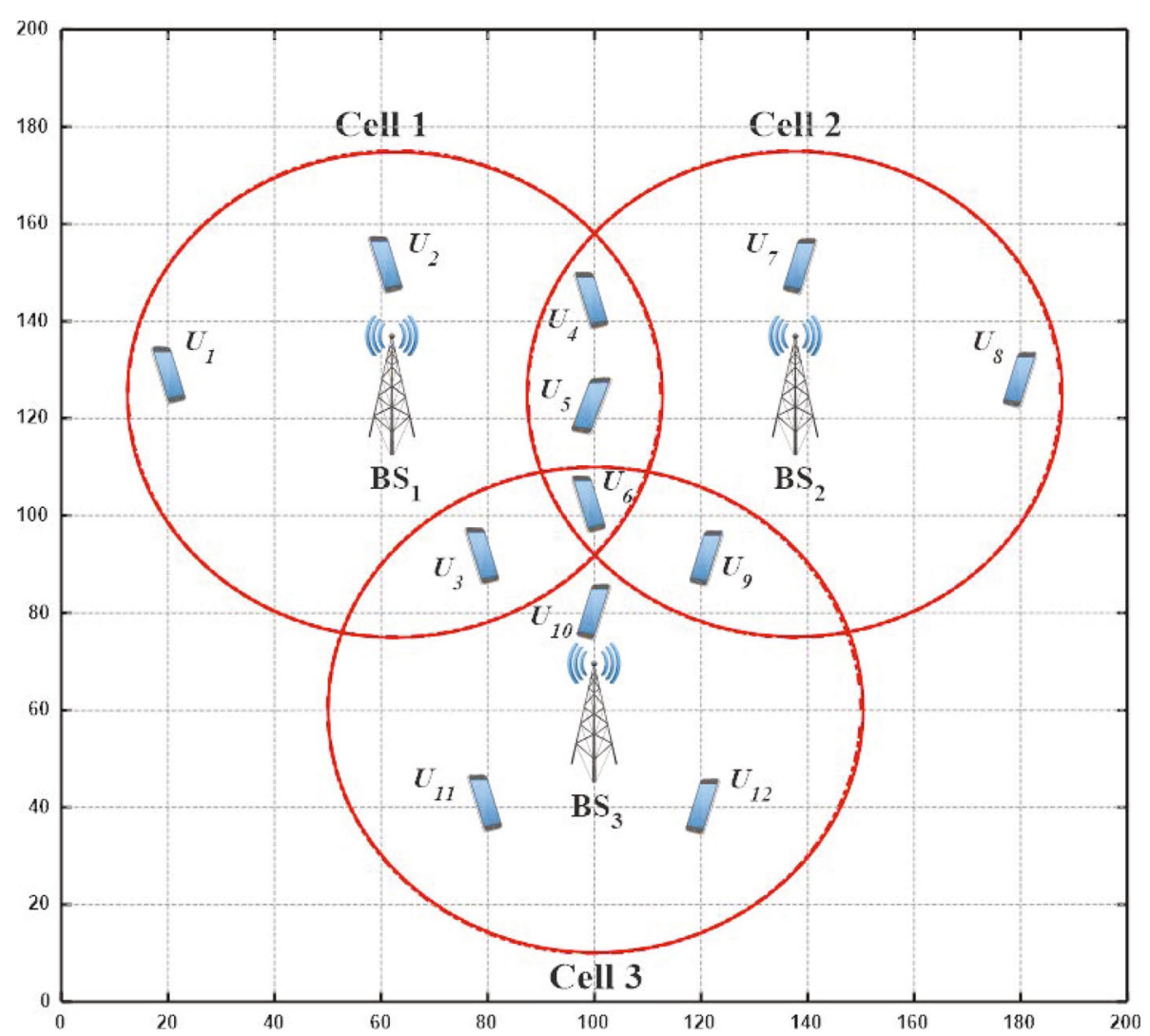

Fig. 2 Simulated network topology

solutions pose a trade-off between complexity, optimality, and stability.

Remark 15 Problem C-J-UA-CA-PA globally optimally maximizes proportional fairness, but does not necessarily ensure that the resulting user association and channel assignment are stable.

The intuition behind Remark 15 is that for some network instance, a certain user may be paired to a certain channel in the solution of problem C-J-UA-CA-PA. However, that (user, channel) pair forms a blocking pair and thus cannot be included in the stable matching solution of any of the stable matching algorithms. In other words, that (user, channel) pair may be formed under problem C-J-UA-CA-PA so as to obtain the global optimal solution, however would be excluded from the matching solution to ensure stability. Specifically, stability is particularly important from a network's perspective, as it ensures that no user or base station would unilaterally want to change its channel assignment or association. This in turn minimizes communications overheads and signaling.

\section{Simulation results}

This section evaluates the performance of the proposed solution procedure and stable matching algorithms. The simulations assume a network consisting of $Q=3$ base stations/cells, with $N=12$ users, $K=9$ channels in a $200 \times 200 \mathrm{~m}^{2}$ area and cell radius of $50 \mathrm{~m}$ per base station (see Fig. 2). Also, each base station $\mathrm{BS}_{q} \in \mathcal{B}$ for $q \in\{1,2,3\}$ is allocated the channel sets $\mathcal{C}_{1}=\left\{C_{1}, C_{2}, C_{3}\right\}, \mathcal{C}_{2}=$ $\left\{C_{4}, C_{5}, C_{6}\right\}$, and $\mathcal{C}_{3}=\left\{C_{7}, C_{8}, C_{9}\right\}$, respectively. Moreover, the transmit power per allocated channel is set to $P=1 \mathrm{~W}$, the noise variance is $N_{0}=10^{-7} \mathrm{~W}$, and the path loss exponent is $v=3$. The target minimum SINR per user is set to $\gamma_{T_{n}}=\gamma_{T}=15 \mathrm{~dB}, \forall U_{n} \in \mathcal{U}$. Furthermore, the simulations are averaged over $10^{3}$ independent instances of randomly generated channel coefficients, where the channel coefficients are assumed to be quasi-static during each network instance, but vary from one network instance to another ${ }^{8}$.

In the simulations, the following scenarios are compared:

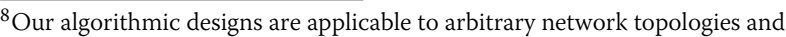
sets of parameters, provided that the selected parameters yield feasible solutions.
} 


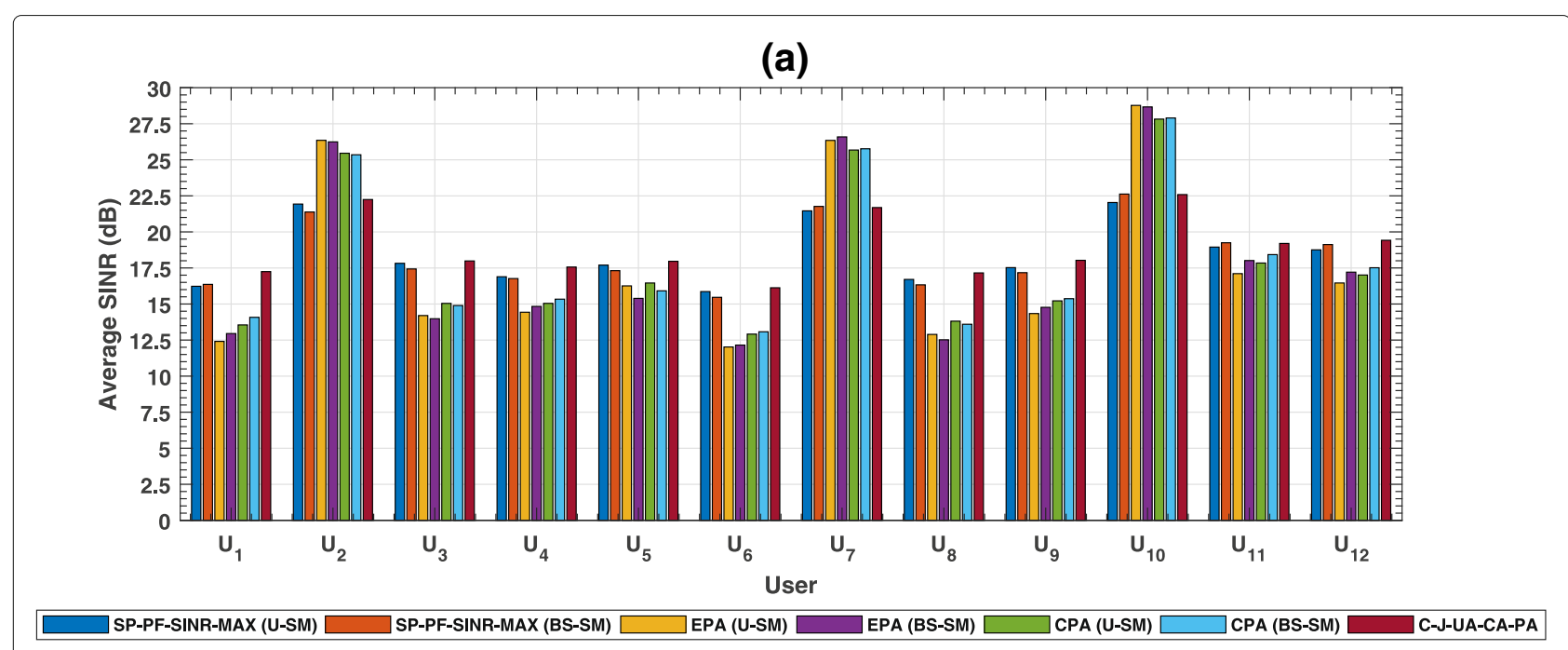

(b)

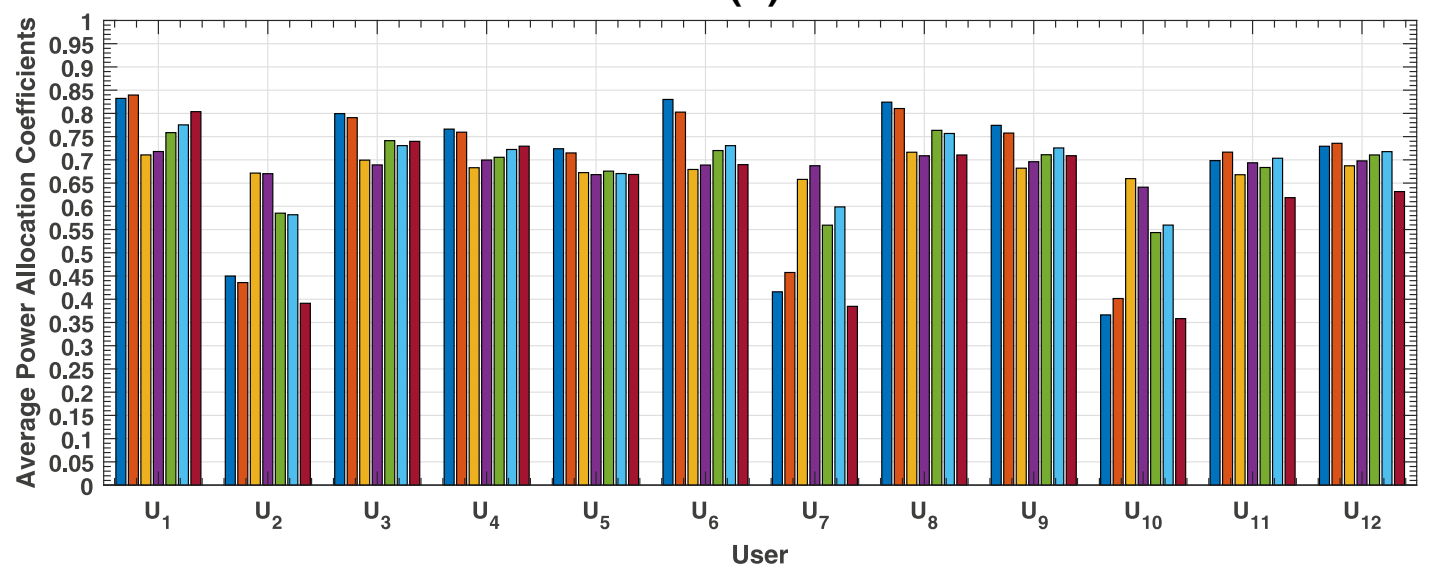

Fig. 3 a Average SINR (dB) and $\mathbf{b}$ average power allocation coefficients - Scenario 1

Scenario 1: $\xi_{q}=4$ and $\zeta_{q, k}=2, \forall C_{q, k} \in \mathcal{C}_{q}$ and $\forall \mathrm{BS}_{q} \in \mathcal{B}$. Scenario 2: $\xi_{q}=4$ and $\zeta_{q, k}=3, \forall C_{q, k} \in \mathcal{C}_{q}$ and $\forall B S_{q} \in \mathcal{B}$.

These scenarios aim at investigating the effect of assigning different numbers of users to each channel. In addition, in the aforementioned scenarios, the following two deterministic power allocation schemes are compared:

\section{Equal power allocation (EPA)}

In this scheme, the power is equally allocated across all users over each channel. Specifically, for Scenario 1 (i.e., $\zeta_{q, k}=2$ ), $a_{q, n}^{k}=1 / 2$, while in Scenario 2 (i.e., $\left.\zeta_{q, k}=3\right), a_{q, n}^{k}=1 / 3, \forall U_{n} \in \mathcal{U}_{q}, \forall C_{q, k} \in \mathcal{C}_{q}$, and $\forall B S_{q} \in \mathcal{B}$.

\section{Conventional power allocation (CPA)}

For this scheme, the power allocation coefficients of the ordered network users (for $1 \leq n \leq \zeta_{q, k}$ ) are set as $a_{q, n}^{k}=\frac{3-n}{3}$ in Scenario 1 , while in Scenario 2, $a_{q, n}^{k}=\frac{4-n}{6}, \forall U_{n} \in \mathcal{U}_{q}, \forall C_{q, k} \in \mathcal{C}_{q}$, and $\forall B S_{q} \in \mathcal{B}$ [34]. That is, for the ordered users under Scenario 1, $a_{q, 1}^{k}=\frac{2}{3}$ and $a_{q, 2}^{k}=\frac{1}{3}$ (i.e., $\left.a_{q, 1}^{k}>a_{q, 2}^{k}\right)$, while under Scenario 2, $a_{q, 1}^{k}=\frac{3}{6}, a_{q, 2}^{k}=\frac{2}{6}$, and $a_{q, 3}^{k}=\frac{1}{6}\left(\right.$ i.e., $\left.a_{q, 1}^{k}>a_{q, 2}^{k}>a_{q, 3}^{k}\right)$.

For notational convenience, let the proposed $U$-SM and $B S-S M$ matching algorithm when combined with the SP-PF-SINR-MAX be denoted SP-PF-SINR-MAX (U-SM) and SP-PF-SINR-MAX (BS-SM) for short. Similarly, let the power allocation schemes when combined with the proposed stable matching algorithms be denoted $E P A$ (U-SM), EPA (BS-SM), CPA (U-SM), and CPA (BS-SM). Lastly, the proposed algorithms are compared to the $C-J$ UA-CA-PA scheme ${ }^{9}$.

In Fig. 3a, the average SINR per user for Scenario 1 is illustrated. In particular, it can be seen that for the $S P$ -

\footnotetext{
${ }^{9}$ The C-J-UA-CA-PA problem is solved via MIDACO [35], with tolerance set to 0.001 . Moreover, problem C-J-UA-CA-PA involves a total of 648 decision variables and 144 inequality constraints.
} 
PF-SINR-MAX (U-SM) and SP-PF-SINR-MAX (BS-SM) algorithms, all network users satisfy the target minimum SINR per user of $\gamma_{T}=15 \mathrm{~dB}$. On the other hand, for the proposed stable matching algorithms with the EPA and $C P A$ schemes, not all users satisfy $\gamma_{T}$. This is due to the fact that power is deterministically allocated to the paired users and without ensuring that the target minimum SINR constraint per user over each channel is satisfied. This proves that the $S P-P F-S I N R-M A X$ is effective in guaranteeing that $\gamma_{T}$ is met for all network users. It is also evident that the highest average SINR among all users is achieved by users $U_{2}, U_{7}$, and $U_{10}$, which is due to their locations being closest to their respective base stations, as can be seen from Fig. 2. Moreover, the C-J-UA-CA-PA scheme marginally outperforms the proposed SP-PF-SINR-MAX $(U$-SM) and SP-PF-SINR-MAX (BS-SM) algorithms, while guaranteeing that $\gamma_{T}$ is achieved. This is because the $C$ $J-U A-C A-P A$ scheme maximizes proportional fairness of the SINR of all network users via user association and channel assignment without any bearing on the network stability (as per Remark 15). Figure 3b shows the average power allocation coefficient per user. Evidently, the $S P$ PF-SINR-MAX (U-SM) and SP-PF-SINR-MAX (BS-SM) algorithms allocate power very similarly to all network users. However, the power allocated per user resulting from the C-J-UA-CA-PA scheme is lower than the aforementioned algorithms. As before, this is attributed to the fact that the C-J-UA-CA-PA scheme aims at maximizing proportional fairness without necessarily enforcing stability. Generally speaking, the $C-J-U A-C A-P A$ scheme achieves only marginally higher average SINR per network user with relatively lower transmit power per user. Lastly, it is observed that the users $U_{2}, U_{7}$, and $U_{10}$ are allocated relatively lower power than the other network users, since they are relatively closer to their respective base stations than the other users (i.e., in agreement with the concept of NOMA).

Similar observations to Fig. 3 can be made for Scenario 2 (see Fig. 4a and b). However, the average SINR per user in Scenario 2 is relatively lower than in Scenario 1.

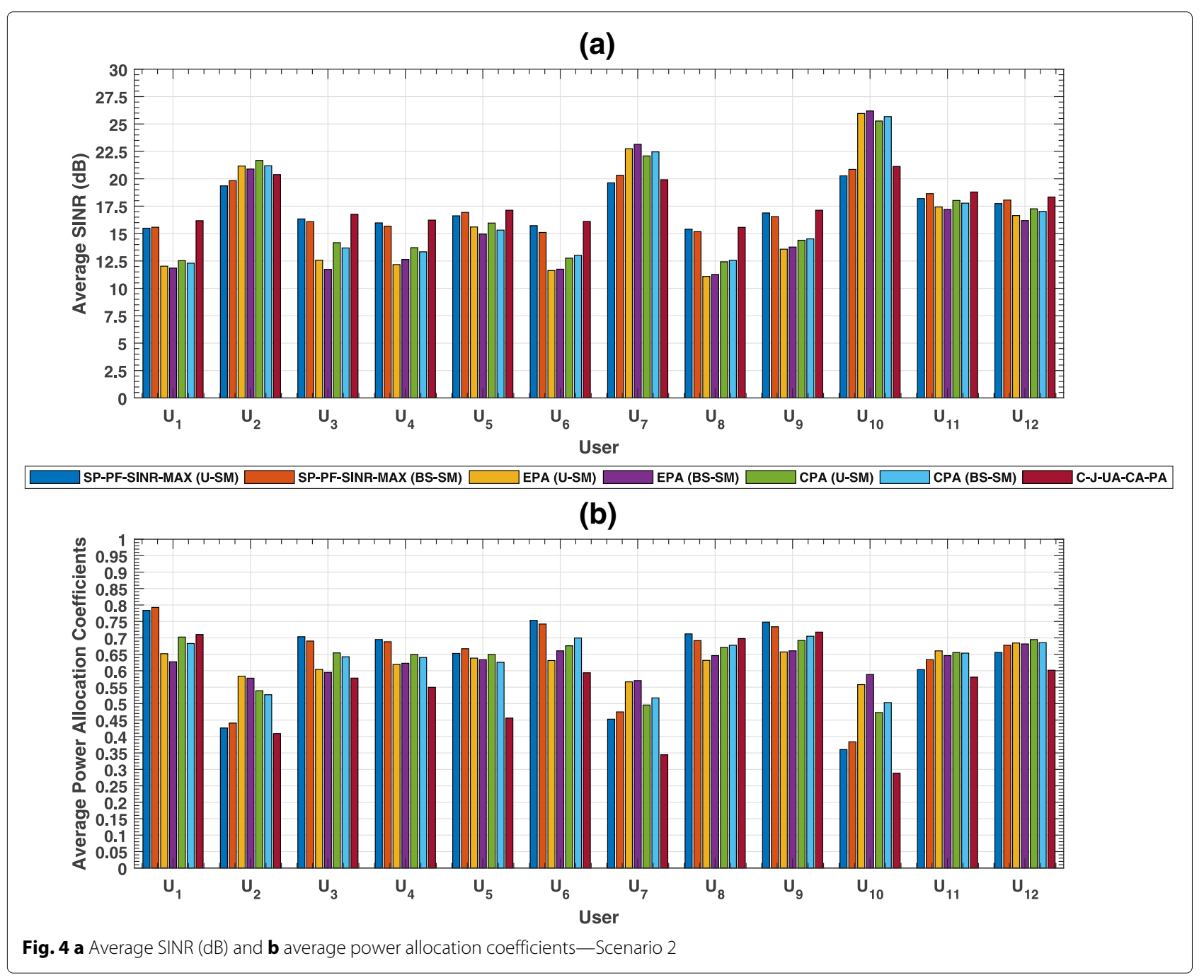


This is attributed to the fact that in Scenario 2, $\zeta_{q, k}=$ $3, \forall C_{q, k} \in \mathcal{C}_{q}, \forall B S_{q} \in \mathcal{B}$, which implies more users can be assigned to each channel. This in turn means that the available transmit power over each channel is distributed over a potentially larger number of users, and hence, each user's share of the allocated power is less than that of Scenario 1. As before, it can be seen from Fig. 4a that $\gamma_{T}$ is satisfied for all network users under both proposed SP-PF-SINR-MAX (U-SM) and SP-PF-SINR-MAX (BS$S M)$ algorithms as well as the C-J-UA-CA-PA scheme, but this is not the case for the EPA and CPA schemes when combined with the $U-S M$ and $B S-S M$ matching algorithms.

Figure 5 illustrates Jain's fairness index based on the average SINR of each network user under both scenarios [36]. Evidently, under both scenarios, the C-J-UA-CA-PA scheme achieves the greatest fairness, which is followed by the proposed SP-PF-SINR-MAX (U-SM) and SP-PFSINR-MAX (BS-SM) algorithms, respectively. Contrarily, the EPA and CPA schemes when combined with the $U-S M$ and $B S-S M$ matching algorithms achieve the lowest fairness among all schemes. In addition, the different schemes achieve relatively greater fairness in Scenario 2 than in Scenario 1. This is because Scenario 2 considers a potentially greater number of user combinations over each channel, which in turn maximizes proportional fairness across a greater number of users per channel. Hence, this confirms that the $S P-P F-S I N R-M A X$ maximizes proportional fairness among all network users.

In Fig. 6, the average number of associated users per base station of the SP-PF-SINR-MAX (U-SM) algorithm is illustrated. One can see that for Scenario 1 (2), base station $\mathrm{BS}_{3}$ is associated with 4 users about $90.79 \%$ (93.47\%) of the time, which is greater than base stations $\mathrm{BS}_{1}$ and $\mathrm{BS}_{2}$. More importantly, $\mathrm{BS}_{3}$ is always associated with at least 3 users under both scenarios. In Fig. 7, the average number of associated users per base station of the SP-PF-SINR$M A X(B S-S M)$ is illustrated. As before, for $\mathrm{BS}_{3}$, the average numbers of associated users for Scenarios 1 and 2 are $82.88 \%$ and $84.07 \%$, respectively, which are greater than those of $\mathrm{BS}_{1}$ and $\mathrm{BS}_{2}$. By comparing Figs. 6 and 7, base stations $\mathrm{BS}_{1}$ and $\mathrm{BS}_{2}$ under the SP-PF-SINR-MAX (BS$S M)$ algorithm are associated with four users more often than the SP-PF-SINR-MAX (U-SM) algorithm, under both scenarios. An opposite observation can be made for base station $\mathrm{BS}_{3}$.

Figure 8 illustrates the percentage of user unassignment under the $U-S M$ and $B S-S M$ algorithms when combined with the SP-PF-SINR-MAX for both scenarios. Evidently, $U_{1}$ and $U_{8}$ are the two users with the highest percentage of unassignment, and this is because they are farthest from their respective base stations as well as not being in the overlapping region of other base stations. It is also noteworthy that although user $U_{6}$ is located at the cell edge of all three base stations, the percentage of it being unassigned is at most $2 \%$, under both scenarios. This is due to the fact that it can be allocated a channel by any of the three base stations, since it falls within the overlapping region of all three base stations. Additionally, all users except $U_{1}$ and $U_{8}$ are assigned to a channel and associated with a base station at least $90 \%$ of the time, under both scenarios.

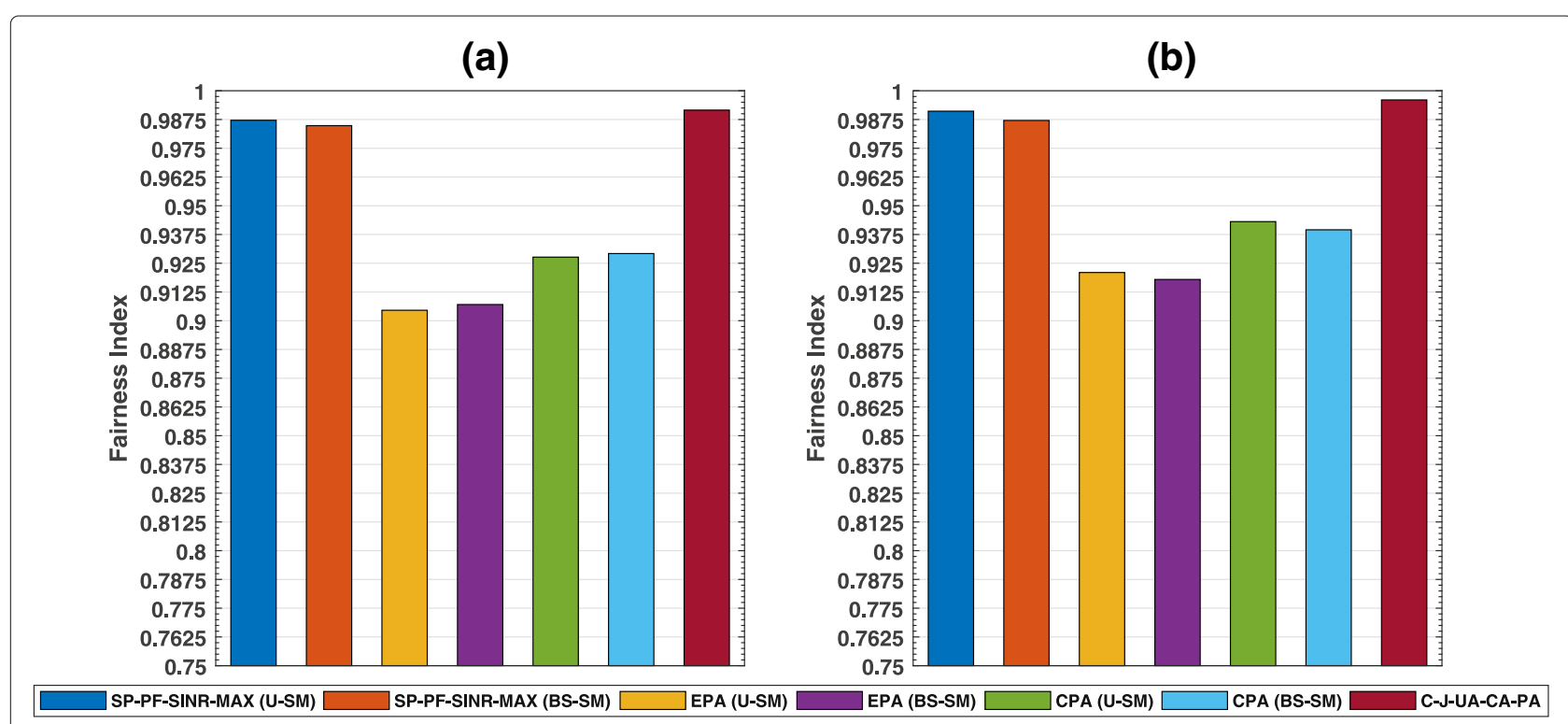

Fig. 5 Jain's fairness index: a Scenario 1 and $\mathbf{b}$ Scenario 2 


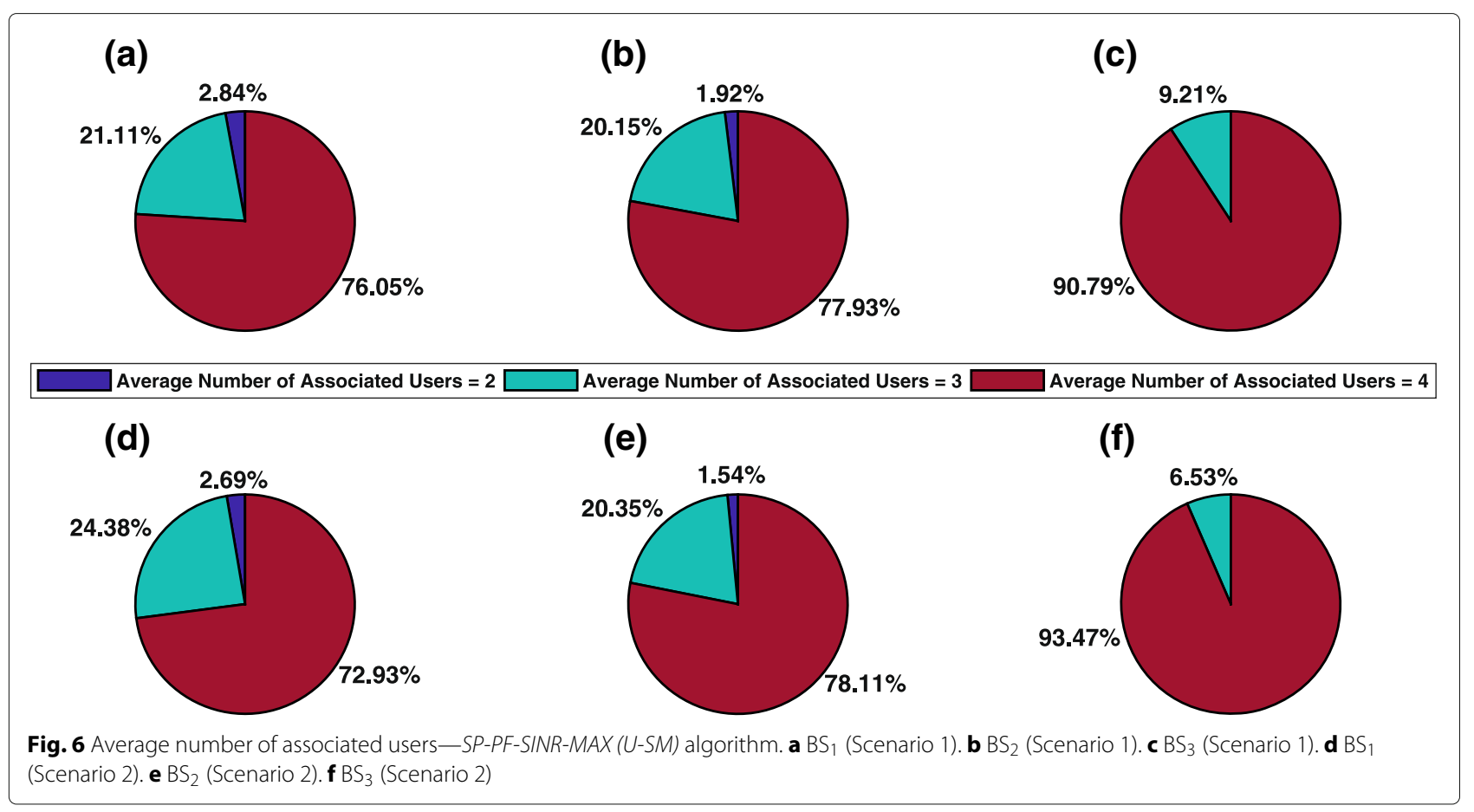

Figure 9 demonstrates the percentage of channel assignment of users $U_{3}, U_{4}, U_{5}, U_{6}$, and $U_{9}$ under both matching algorithms (with SP-PF-SINR-MAX) under Scenario 1. Specifically, one can see that user $U_{3}$ is paired only with the channels of base stations $\mathrm{BS}_{1}$ and $\mathrm{BS}_{3}$ (i.e., in $\mathcal{C}_{1}$ and $\left.\mathcal{C}_{3}\right)$. As for user $U_{6}$, it is paired with all channels, since it falls within the overlapping region of all three base stations. Similar observations can be made to the other users. To summarize, it has been verified that all users can only be paired with the channels corresponding to cells

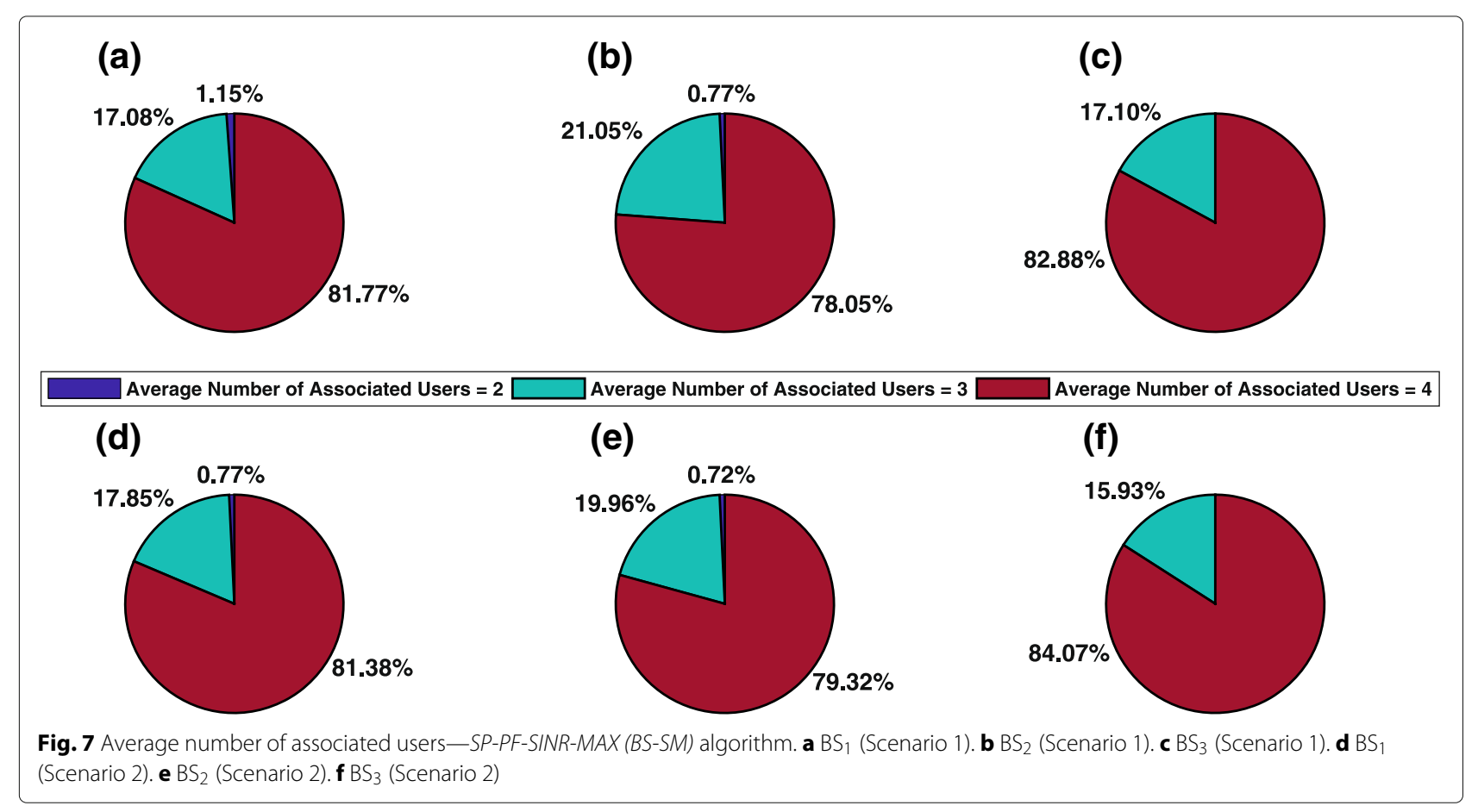




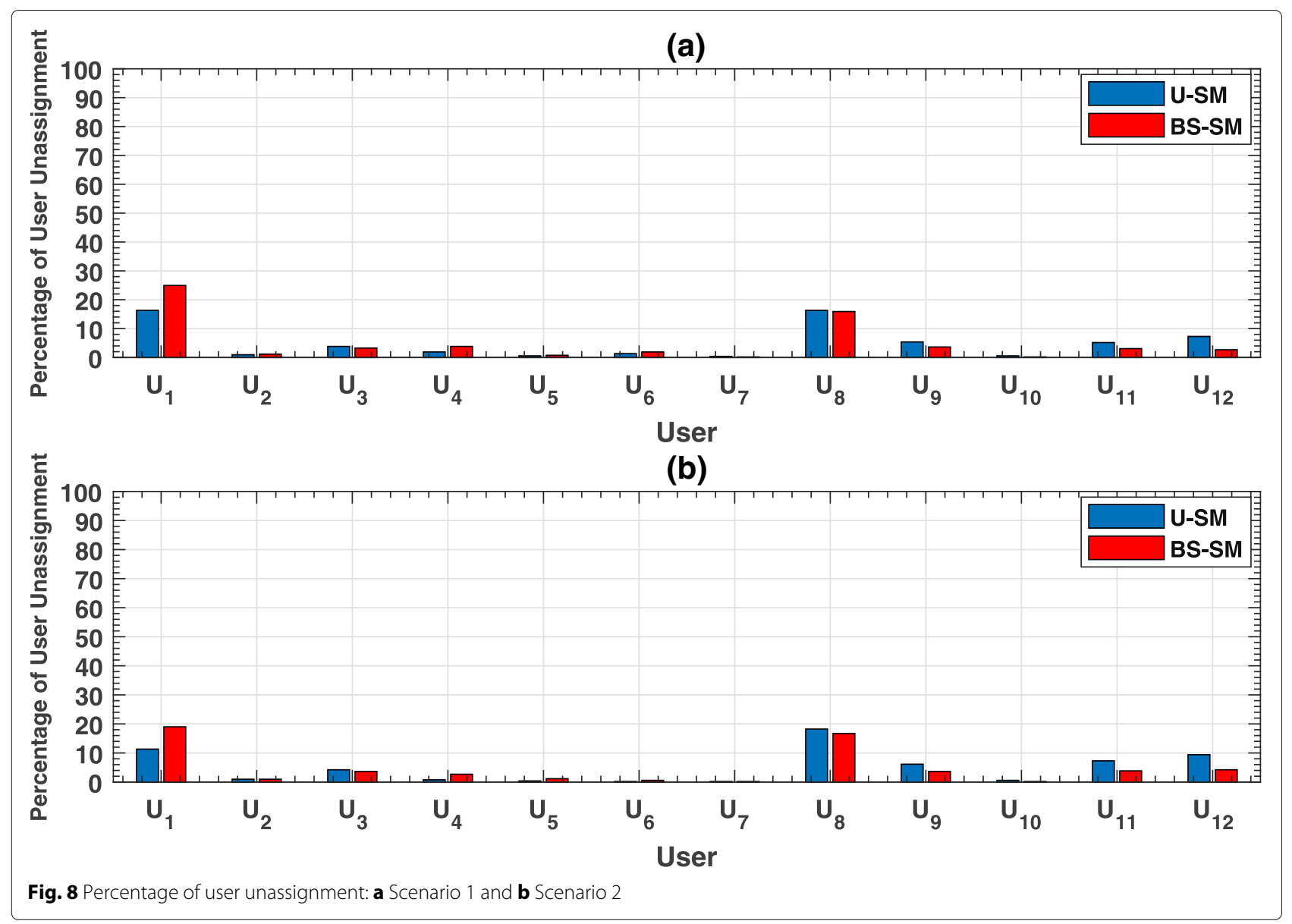

where they are located. Similar observations are made for Scenario 2 in Fig. 10.

Figure 11a and $\mathrm{b}$ illustrate the average number of iterations of each stable matching algorithms (with SP-PFSINR-MAX) under Scenarios 1 and 2. It is evident that both algorithms require almost the same number of iterations under the two scenarios. More importantly, the $B S-S M$ algorithm requires more iterations than its $U$ $S M$ counterpart algorithm. In addition, for our simulated network scenario in Fig. 2, $\left|\mathcal{C}_{1}\right|=\left|\mathcal{C}_{2}\right|=\left|\mathcal{C}_{3}\right|=3$, and $\left|\mathcal{U}_{1}\right|=\left|\mathcal{U}_{2}\right|=\left|\mathcal{U}_{3}\right|=6$. Based on Remarks 5, 8, and 13, and for Scenario 1, the execution of the solution procedure locally at each base station requires a total of 63 iterations, of which 45 iterations involve the solution of a convex optimization problem, which can be solved within polynomial-time complexity. As for scenario 2, each base station involves at total of 123 iterations of the solution procedure, of which 105 iterations involve the solution a convex optimization problem. In turn, both SP-PF-SINR-MAX (U-SM) and SP-PF-SINR$M A X(B S-S M)$ algorithms can be executed efficiently and with lower computational complexity than the C-J-UA-
$C A-P A$ scheme. On the other hand, in Fig. 11c, the percentage of identical stable matchings of the proposed stable matching algorithms is illustrated. Particularly, it can be seen that for Scenario 2, the percentage of identical matchings is slightly less than that of Scenario 1. This is due to the fact in Scenario 2, there are more user combinations than in the case of Scenario 1, which results in identical matchings occurring slightly less often. In all, increasing the maximum number of users that can be assigned a channel reduces the possibility of having identical matchings for both stable matching algorithms. This also explains the discrepancies in the resulting average SINR, average number of associated users with each base station, and percentages of user unassignment and channel assignment of the proposed stable matching algorithms.

In summary, the proposed algorithmic solutions have been shown to yield comparable performance to the $C$ $J-U A-C A-P A$ scheme in terms of average SINR per user and network SINR-based fairness. Additionally, the proposed algorithms have been shown to efficiently associate users with base stations and assign them to channels. 


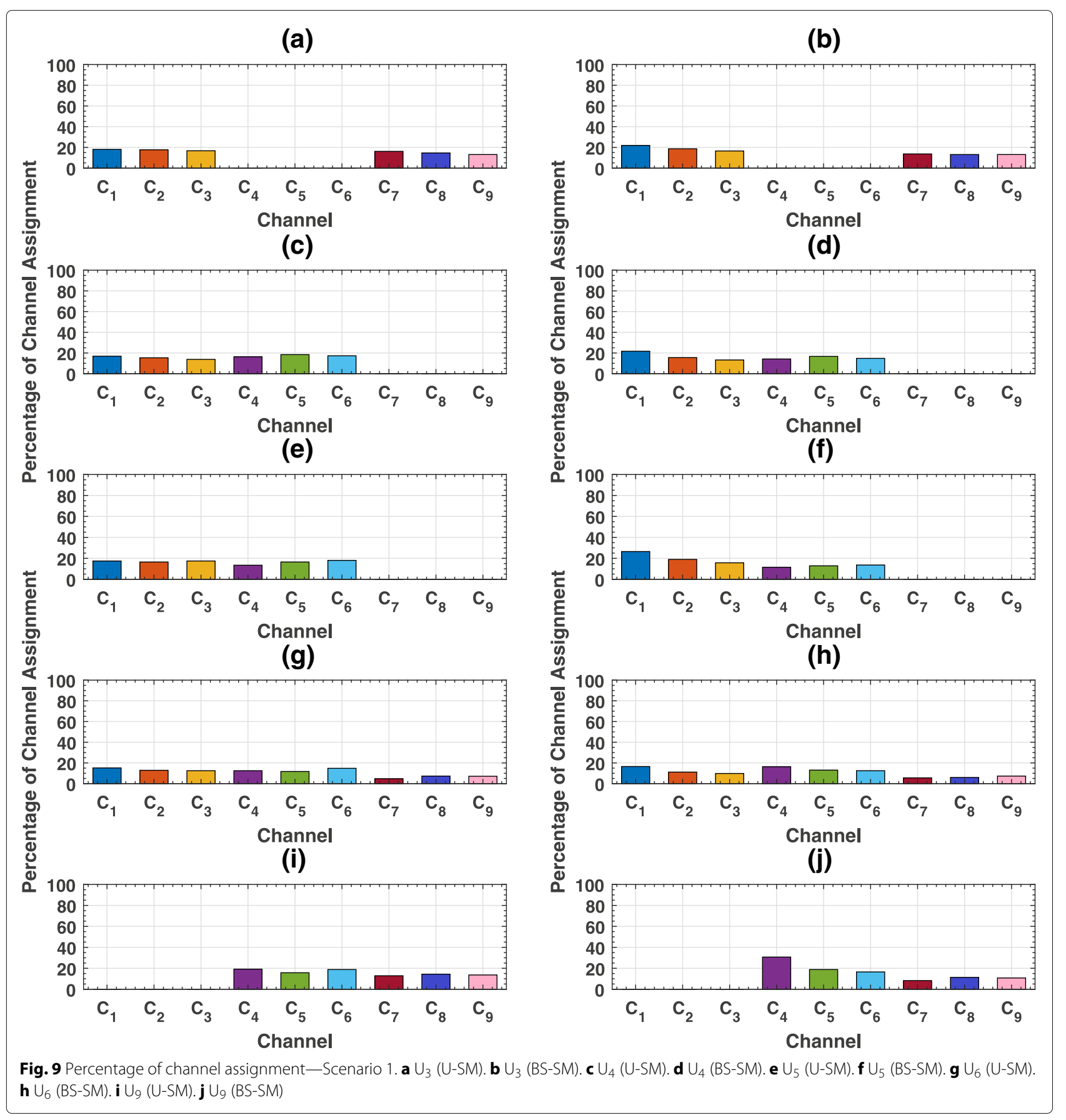

More importantly, the proposed solution procedure can be executed locally at each base station to determine the proportional fairness maximizing power allocation and preference lists, while the stable matching algorithms can be efficiently executed among the base stations to perform user association and channel assignment with minimal communication overheads and signaling, and with the added merit of network stability. In other words, the proposed SP-PF-SINR-MAX (U-SM) and SPPF-SINR-MAX (BS-SM) algorithms offer a reasonable trade-off between average SINR, fairness, complexity, and stability.

\section{Conclusions}

In this paper, the problem of joint user association and channel assignment with proportional fairness 


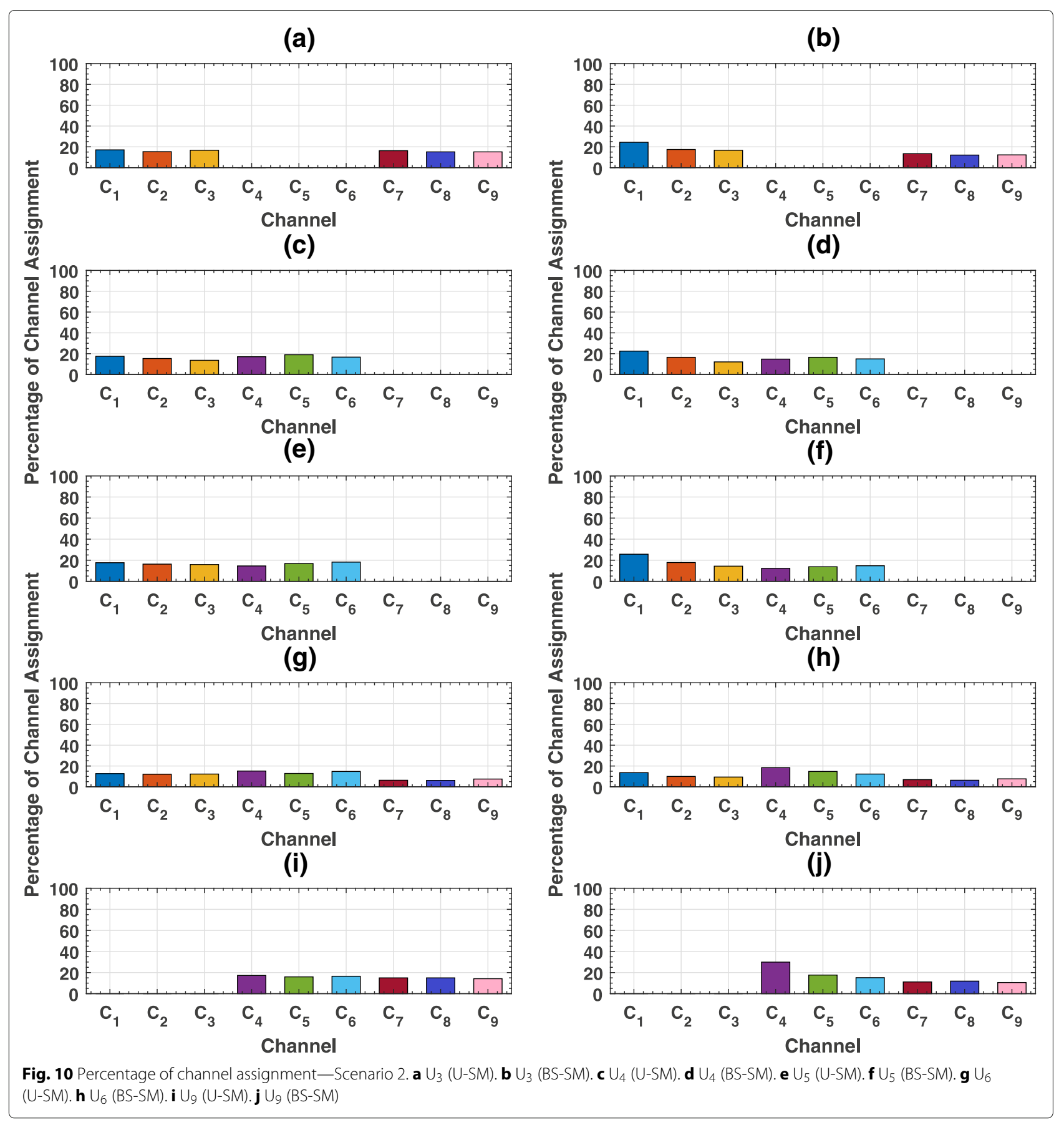

SINR-based power allocation in downlink multi-cell NOMA networks has been studied. Particularly, a lowcomplexity iterative solution procedure has been devised to determine the optimal power allocation for proportional fairness SINR-based maximization over each channel within each cell. Moreover, two many-to-one polynomial-time complexity matching algorithms have been proposed to associate users with base stations and perform channel assignment. To validate the efficacy of the proposed solution procedure and stable matching algorithms, extensive simulation results have been presented, which illustrate that the proposed algorithms efficiently yield comparable SINR per user to the C-J-UA-CA-PA scheme as well as maximizing proportional fairness and satisfying QoS constraints. Finally, the proposed algorithms have been shown to 


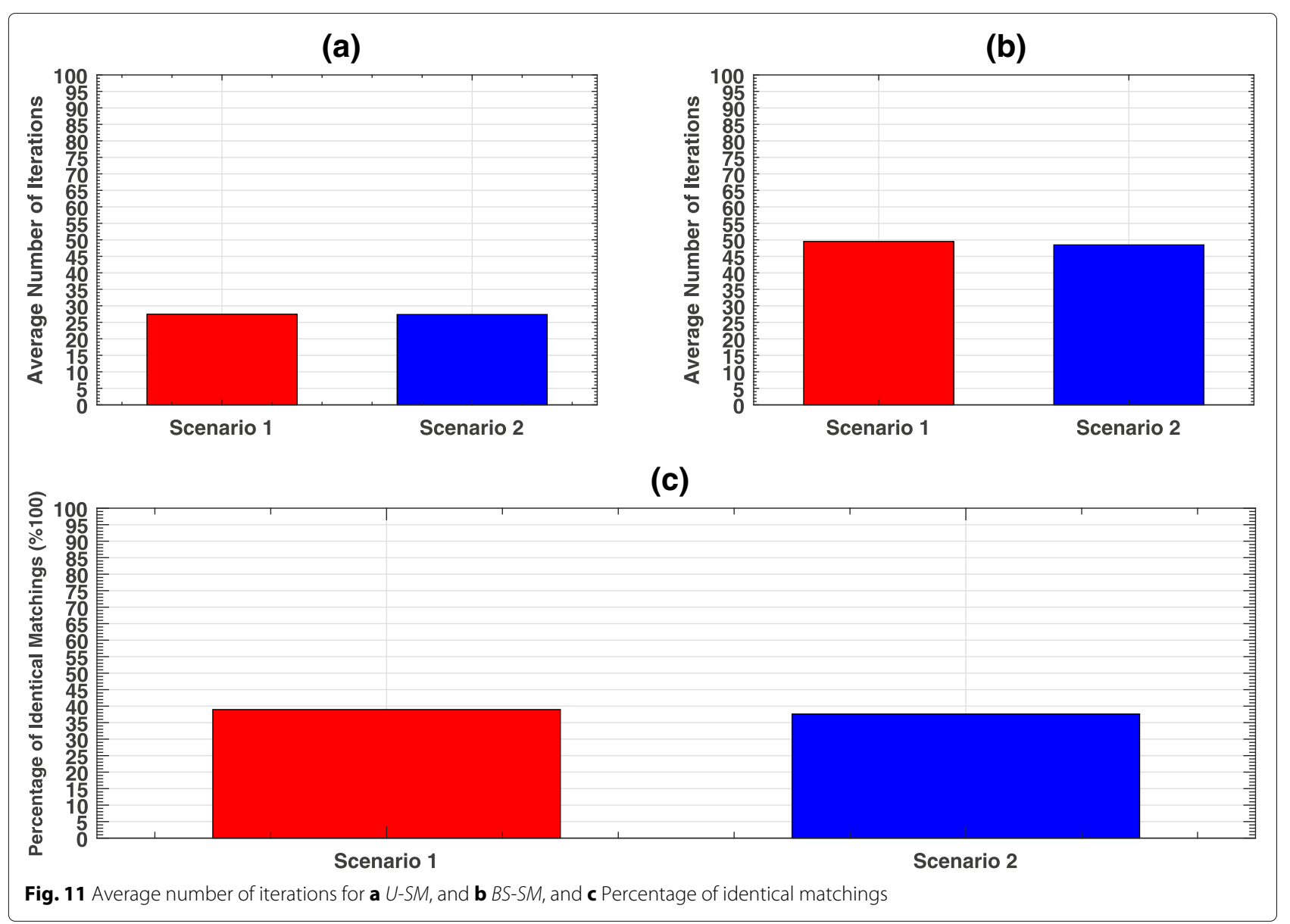

efficiently assign channels to cell-edge users and especially those within the overlapping region of multiple cells.

\section{Abbreviations}

BS: Base station; BS-SM: Base station-oriented stable matching; C-J-UA-CA-PA Centralized joint user association, channel assignment, and power allocation; CPA: Conventional power allocation; CoMP: Coordinated multipoint; CSI: Channel state information; CSIT: Channel state information at transmitter; D2D: Device-to-device; EPA: Equal power allocation; LF: Linear-fractional; mmWave: Millimeter-wave; NOMA: Non-orthogonal multiple access; OFDMA: Orthogonal frequency-division multiple access; OMA: Orthogonal multiple access; PR-SINR-MAX: Proportional fairness SINR-based maximization; SIC: Successive interference cancelation; SINR: Signal-to-interference-plus-noise ratio; SP-PF-SINR-MAX: Solution procedure for proportional fairness SINR maximization; SPA: Student-project allocation; SPM: Sum-power minimization; SRM: Sum-rate maximization; U-SM: User-oriented stable matching

\section{Acknowledgements}

Not applicable.

\section{Authors' contributions}

All authors have contributed to the work presented in this paper. Particularly, the contributions can be stated as follows: MWB contributed to the problem formulation, mathematical analysis, writing, and simulation. ZB contributed to the mathematical analysis and simulation. EA contributed to the problem formulation, mathematical analysis, and writing. Lastly, all authors read and approved the final manuscript.

\section{Funding}

This work is supported partially by the Kuwait Foundation for the Advancement of Sciences (KFAS), under project code PN17-15EE-02.

\section{Competing interests}

The authors declare that they have no competing interests.

\section{Author details}

${ }^{1}$ Department of Electrical Engineering, College of Engineering and Petroleum, Kuwait University, Kuwait City, Kuwait. ' ${ }^{2}$ Department of Computer Engineering, College of Engineering and Petroleum, Kuwait University, Kuwait City, Kuwait. ${ }^{3}$ School of Electrical and Electronic Engineering, University of Manchester, Manchester, UK.

Received: 31 August 2018 Accepted: 25 July 2019

Published online: 03 September 2019

\section{References}

1. S. M. R. Islam, N. Avazov, O. A. Dobre, K. S. Kwak, Power-domain non-orthogonal multiple access (NOMA) in 5 G systems: potentials and challenges. IEEE Commun. Surv. Tutor. 19(2), 721-742 (2017)

2. L. Dai, B. Wang, Y. Yuan, S. Han, C.-L. I, Z. Wang, Non-orthogonal multiple access for 5G: solutions, challenges, opportunities, and future research trends. IEEE Commun. Mag. 53(9), 74-81 (2015)

3. P. Xu, K. Cumanan, Optimal power allocation scheme for non-orthogonal multiple access with $\alpha$-fairness. IEEE J. Sel. Areas Commun. 35(10), 2357-2369 (2017)

4. M. B. Shahab, M. Irfan, M. F. Kader, S. Y. Shin, User pairing schemes for capacity maximization in non-orthogonal multiple access systems. Wirel. Commun. Mob. Comput. 16(17), 2884-2894 (2016) 
5. Z. Yang, Z. Ding, P. Fan, N. Al-Dhahir, A general power allocation scheme to guarantee quality of service in downlink and uplink NOMA systems. IEEE Trans. Wirel. Commun. 15(11), 7244-7257 (2016)

6. J. Zhu, J. Wang, Y. Huang, S. He, X. You, L. Yang, On optimal power allocation for downlink non-orthogonal multiple access systems. IEEE J. Sel. Areas Commun. 35(15), 2744-2757 (2017)

7. W. Shin, M. Vaezi, B. Lee, D. J. Love, J. Lee, H. V. Poor, Non-orthogonal multiple access in multi-cell networks: theory, performance, and practical challenges. IEEE Commun. Mag. 55(10), 176-183 (2017)

8. Y. Fu, Y. Chen, C. W. Sung, Distributed power control for the downlink of multi-cell NOMA systems. IEEE Trans. Wirel. Commun. 16(9), 6207-6220 (2017)

9. Z. Yang, C. Pan, W. Xu, Y. Pan, M. Chen, M. Elkashlan, Power control for multi-cell networks with non-orthogonal multiple access. IEEE Trans. Wirel. Commun. 17(2), 927-942 (2018)

10. L. You, D. Yuan, L. Lei, S. Sun, S. Chatzinotas, B. Otersten, Resource optimization with load coupling in multi-cell NOMA. IEEE Trans. Wirel. Commun. 17(7), 4735-4749 (2018)

11. M. Ali, E. Hossain, D. I. Kim, Coordinated multipoint transmission in downlink multi-cell NOMA systems: models and spectral efficiency performance. IEEE Wirel. Commun. 25(2), 24-31 (2018)

12. W. U. Khan, Z. Yu, S. Yu, G. A. S. Sidhu, J. Liu, Efficient power allocation in downlink multi-cell multi-user NOMA networks. IET Commun. 13(4), 396-402 (2019)

13. Y.Sun, Z. Ding, X. Dai, On the performance of downlink NOMA in multi-cell mmWave networks. IEEE Commun. Lett. 22(11), 2366-2369 (2018)

14. D. J. Abraham, R. W. Irving, D. F. Manlove, The student-project allocation problem. Int. Symp. Algoritm. Comput. (ISAAC) - Lect. Notes Comput. Sci. 2906, 474-484 (2003)

15. D. J. Abraham, R. W. Irving, D. F. Manlove, Two algorithms for the student-project allocation problem. J. Discret. Algoritm. 5(1), 73-90 (2007)

16. W. Liang, Z. Ding, L. Song, User pairing for downlink non-orthogonal multiple access networks using matching algorithm. IEEE Trans. Commun. 65(12), 5319-5332 (2017)

17. B. Di, L. Song, Y. Li, Sub-channel assignment, power allocation and user scheduling for non-orthogonal multiple access networks. IEEE Trans. Wirel. Commun. 15(11), 7686-7698 (2016)

18. J. Zhao, Y. Liu, K. K. Chai, Y. Chen, M. Elkashlan, Joint subchannel and power allocation for NOMA enhanced D2D communications. IEEE Trans. Commun. 65(11), 5081-5094 (2017)

19. J. Zhao, Y. Li, K. K. Chai, A. Nallanathan, Y. Chen, Z. Han, Spectrum allocation and power control for non-orthogonal mutliple access in HetNets. IEEE Trans. Wirel. Commun. 16(9), 5825-5837 (2017)

20. X. Su, H. Yu, W. Kim, C. Choi, D. Choi, Interference cancellation for non-orthogonal multiple access used in future wireless mobile networks. EURASIP J. Wirel. Commun. Netw. 231, 1-12 (2016). https://doi.org/10. 1186/s13638-016-0732-z

21. H. Arsham, A. B. Kahn, A complete algorithm for linear fractional programs. Comput. Math. Appl. 20(7), 11-23 (1990)

22. L. Nowak, Relaxation and decomposition methods for mixed integer nonlinear programming. (Spring Science and Business Media, Birkhauser Verlag - Basel, 2005)

23. P. Bonami, M. Kilinc, J. Linderoth, Algorithms and software for convex mixed integer nonlinear programs. IMA Vol. Math. Appl. 154, 1-39 (2012)

24. D. Yue, G. Guillen-Gosalbez, F. You, Global optimization of large-scale mixed integer linear fractional programming problems: a reformulation-linearization method and process scheduling applications. AlChE J. - Proc. Syst. Eng. 59(11), 4255-4272 (2013)

25. P. D. Tao, L. T. H. An, Convex analysis approach to DC programming theory, algorithms and applications. ACTA Math. Vietnamica. 22(1), 289-355 (1997)

26. A. Alvarado, G. Scutari, J.-S. Pang, A new decomposition method for multiuser DC-programmig and its applications. IEEE Trans. Signal Process. 62(11), 2984-2998 (2014)

27. N. Vucic, S. Shi, M. Schubert, in Proc of IEEE International Symposium on Modeling and Optimization in Mobile, Ad Hoc, and Wireless Networks. DC programming approach for resource allocation in wireless networks (IEEE, Avignon, 2010), pp. 380-386

28. R. Horst, H. Tuy, Global optimization: deterministic approaches. (Springer, Berlin, 1990)
29. R. Horst, T. Q. Phong, N. V. Thoai, J. de Vries, On solving a DC programming problem by a sequence of linear programs. J. Glob. Optim. 1(2), 186-203 (1991)

30. H. Tuy, Global minimization of a difference of two convex functions. Math Program. Study. 30, 150-182 (1987)

31. S. Boyd, L. Vandenberghe, Convex optimization. (Cambridge University Press, Cambridge, 2003)

32. Q.-T. Vien, T. A. Le, B. Barn, C. V. Phan, Optimising energy efficiency of non-orthogonal multiple access for wireless backhaul in heterogenous cloud radio access network. IET Commun. 10(18), 2516-2524 (2016)

33. H. Q. Tran, P. Q. Truong, C. V. Phan, Q.-T. Vien, in Proc of International Conference on Recent Advances in Signal Processing, Telecommunications and Computing (SigTelCom). On the energy efficiency of NOMA for wireless backhaul in multitier heterogenous CRAN (IEEE, Da Nang, 2017), pp. 229-234

34. Z. Ding, Z. Yang, P. Fan, H. V. Poor, On the performance of non-orthogona multiple access in $5 \mathrm{G}$ systems with randomly deployed users. IEEE Signal Process. Lett. 21(12), 1501-1505 (2014)

35. M. Schlueter, MIDACO software performance on interplanetary trajectory benchmarks. Adv. Space Res. 54(4), 744-754 (2014)

36. R. Jain, D. M. Chiu, W. Hawe, A quantitative measure of fairness and discrimination for resource allocation in shared computer systems (1984). DEC Research Report TR-301

\section{Publisher's Note}

Springer Nature remains neutral with regard to jurisdictional claims in published maps and institutional affiliations.

\section{Submit your manuscript to a SpringerOpen ${ }^{\circ}$ journal and benefit from:}

- Convenient online submission

- Rigorous peer review

- Open access: articles freely available online

- High visibility within the field

Retaining the copyright to your article

Submit your next manuscript at $>$ springeropen.com 